\title{
DELTA SETS FOR SYMMETRIC NUMERICAL SEMIGROUPS WITH EMBEDDING DIMENSION THREE
}

\author{
P. A. GARCÍA-SÁNCHEZ, D. LLENA, AND A. MOSCARIELLO
}

\begin{abstract}
This work extends the results known for the Delta sets of non-symmetric numerical semigroups with embedding dimension three to the symmetric case. Thus, we have a fast algorithm to compute the Delta set of any embedding dimension three numerical semigroup. Also, as a consequence of these resutls, the sets that can be realized as Delta sets of numerical semigroups of embedding dimension three are fully characterized.
\end{abstract}

\section{INTRODUCTION}

Delta sets (or sets of distances) were first introduced in [13] as a tool to study factorizations in non-unique factorization domains. Since then several authors have studied their properties. In particular, for numerical semigroups, in [3], the firsts results for some special cases of embedding dimension three numerical semigroups are presented. Delta sets for numerical semigroups are eventually periodic as explained in [5], and thus, if a bound for this periodicity is known, the whole Delta set of a numerical semigroup can be computed. The bounds given in [5] were improved in [9]. In [2] a dynamical procedure to compute Delta sets for numerical semigroups is presented, that makes use of the bound given in [9]; this procedure has been implemented in numericalsgps [7]. Recently, a new procedure to compute Delta sets of any affine semigroup using Göbner basis has been presented in [11. Needless to say, all these algorithms and bounds were the consequence of a better (theoretical) understanding of Delta sets.

In [6] it is shown that the maximum of the Delta set of a numerical semigroup is attained in a Betti element of the semigroup (indeed, it is shown that this holds for a wider class of atomic monoids). This does not provide a way to compute the whole set. The minimum was known to be the greatest common divisor of the Delta set since [13]; however, the elements in the interval determined by this minimum and maximum element of the Delta set are not known in general. Some realization results were given in [4], while in [10] the sets that can be realized as the Delta set of a non-symmetric numerical semigroup with embedding dimension three are completely characterized. In that paper, the authors present a procedure that strongly reduces the time needed to compute the Delta sets of non-symmetric embedding dimension three numerical semigroups. In this manuscript, we extend this algorithm to the symmetric case.

Although the main results yield the same algorithm for both symmetric and non-symmetric numerical semigroups with embedding dimension three, there are significant differences in the intermediate results and their proofs. These differences stem from the different structure of the Betti elements of these semigroups (see Proposition 3 ).

The factorizations of the Betti elements of non-symmetric embedding dimension three numerical semigroups have been thoroughly studied, and thus provided an easier starting point. However, in that case, some technical and tedious detours were needed to obtain our result.

Due to the fact that, in the symmetric case, we do not have a unique minimal presentation (in contraposition to what happens in the non-symmetric case), the first step is to choose the right

2010 Mathematics Subject Classification. 05A17,20M13,20M14.

Key words and phrases. Numerical Semigroups, non-unique factorization, Delta set, Euclid's Algorithm.

The first and second author are supported by the project FQM-343, MTM2014-55367-P, and FEDER funds. 
factorization of the Betti elements to start our algorithm. However, in this case we can work directly on the Euclid's set (see Definition [10), whereas this cannot be done in the non-symmetric case without building a certain theoretical framework. In this way, we can better see the idea behind the main result, and the technical results are no longer necessary. Moreover, the proofs and the reading of the paper are more comfortable, and the number of pages is reduced. Unfortunately, from our reasoning, it emerged that there are no relations between these two cases; we think that it is not possible to find a common way to deduce both settings together.

This paper, even though is complementary to [10, is self-contained, and therefore can be read separately from [10]. After some preliminary results, we explain how we can choose the elements $\delta_{1}$ and $\delta_{2}$ needed to start the algorithm. As we mentioned above, this was not needed in the nonsymmetric case, since in that setting the Delta sets of the Betti elements of the numerical semigroup are singletons. Then, we prove our main result, which yields an algorithm that works in the same way as in the non-symmetric case; in particular, notice that Example 20 provided in this paper gives the same Delta set as in [10, Example 38], because it is obtained from the same $\delta_{1}$ and $\delta_{2}$.

\section{Preliminaries}

Let $\mathbb{N}$ be the set of non negative integers. Take $n_{1}, n_{2}, n_{3} \in \mathbb{N}$ with $\operatorname{gcd}\left(n_{1}, n_{2}, n_{3}\right)=1$, and define $S$ as the numerical semigroup minimally generated by $\left\{n_{1}, n_{2}, n_{3}\right\}$, that is,

$$
S=\left\{a_{1} n_{1}+a_{2} n_{2}+a_{3} n_{3} \mid a_{1}, a_{2}, a_{3} \in \mathbb{N}\right\} .
$$

The condition $\operatorname{gcd}\left(n_{1}, n_{2}, n_{3}\right)=1$ is equivalent to say that the set of gaps, $\mathrm{G}(S)=\mathbb{N} \backslash S$, has finitely many elements. The maximum of $\mathbb{Z} \backslash S$ is called the Frobenius number, and we denote it as $\mathrm{F}(S)$.

Definition 1. A numerical semigroup $S$ is symmetric if $x \in \mathbb{Z} \backslash S$ implies $\mathrm{F}(S)-x \in S$.

The reader interested in numerical semigroups may have a look at 14 and for some applications to 1 .

The set of factorizations of $s \in S$ is

$$
\mathbf{Z}(s)=\left\{\left(z_{1}, z_{2}, z_{3}\right) \in \mathbb{N}^{3} \mid z_{1} n_{1}+z_{2} n_{2}+z_{3} n_{3}=s\right\} .
$$

We denote the length of a factorization $\mathbf{z}=\left(z_{1}, z_{2}, z_{3}\right) \in \mathbf{Z}(s)$ as

$$
\ell(\mathbf{z})=z_{1}+z_{2}+z_{3} \text {. }
$$

The set of lengths of $s \in S$ is

$$
\mathrm{L}(s)=\{\ell(\mathbf{z}) \mid \mathbf{z} \in \mathbf{Z}(s)\} .
$$

It is easy to see that $\mathrm{L}(s) \subset[0, s]$, and consequently $\mathrm{L}(s)$ is finite. So, it is of the form $\mathrm{L}(s)=$ $\left\{l_{1}, \ldots, l_{k}\right\}$ for some positive integers $l_{1}<l_{2}<\cdots<l_{k}$. The set

$$
\Delta(s)=\left\{l_{i}-l_{i-1} \mid 2 \leq i \leq k\right\}
$$

is known as the Delta set of $s \in S$ (or set of distances), and the Delta set of $S$ is defined as

$$
\Delta(S)=\cup_{s \in S} \Delta(s) .
$$

Now, we define

$$
M_{S}=\left\{\mathbf{v}=\left(v_{1}, v_{2}, v_{3}\right) \in \mathbb{Z}^{3} \mid v_{1} n_{1}+v_{2} n_{2}+v_{3} n_{3}=0\right\} .
$$

We can extend the $\ell$ function to elements in $M_{S}$ : for $\mathbf{v}=\left(v_{1}, v_{2}, v_{3}\right) \in \mathbb{Z}^{3}$, set

$$
\ell(\mathbf{v})=v_{1}+v_{2}+v_{3} .
$$

Note that $\ell$ is a linear map.

The aim of this paper is to compute the Delta set of $S$ by using Euclid's algorithm with two special elements in this set. These special distances are associated to particular elements of $S$, called the Betti elements. The definition of Betti element relies in the construction of a graph associated to the elements in the semigroup. Let $s \in S$. The graph $\nabla_{s}$ is the graph with vertices $\mathrm{Z}(s)$, the 
set of factorization of $s$, and $\mathbf{z z}^{\prime}$ is an edge if if $\mathbf{z} \cdot \mathbf{z}^{\prime} \neq 0$, that is, there exists a common nonzero coordinate in both factorizations.

Definition 2. An element $s \in S$ is called a Betti element if and only if $\nabla_{s}$ is not connected. The set of Betti elements will be denoted by $\operatorname{Betti}(S)$.

A numerical semigroup with embedding dimension three might have up to three Betti elements. In [14, Example 8.23] it is shown that $\operatorname{Betti}\left(\left\langle n_{1}, n_{2}, n_{3}\right\rangle=\left\{c_{1} n_{1}, c_{2} n_{2}, c_{3} n_{3}\right\}\right.$, where $c_{i}$ is the least positive integer such that $c_{i} n_{i} \in\left\langle n_{j}, n_{k}\right\rangle,\{i, j, k\}=\{1,2,3\}$.

Proposition 3. 14, Chapter 9] Let $S$ an embedding dimension three numerical then $1 \leq \sharp \operatorname{Betti}(S) \leq$ 3. Moreover, $S$ is non-symmetric if and only if $\sharp \operatorname{Betti}(S)=3$.

Example 4. Consider $S=\langle 3,5,7\rangle=\{0,3,5, \rightarrow\}$ (the arrow means that all integers greater than 5 are in the semigroup). In this case, $\operatorname{Betti}(S)=\{10,12,14\}$, and

$$
\begin{aligned}
& \mathrm{Z}(10)=\{(1,0,1),(0,2,0)\}, \quad \mathrm{L}(10)=\{2\}, \quad \Delta(10)=\emptyset, \\
& \mathrm{Z}(12)=\{(4,0,0),(0,1,1)\}, \quad \mathrm{L}(12)=\{2,4\}, \Delta(12)=\{2\}, \\
& \mathrm{Z}(14)=\{(3,1,0),(0,0,2)\}, \quad \mathrm{L}(14)=\{2,4\}, \Delta(14)=\{2\} .
\end{aligned}
$$

Let $S=\langle 6,8,11\rangle$, with Betti elements 22 and 24 .

$$
\begin{aligned}
& \mathrm{Z}(22)=\{(1,2,0),(0,0,2)\}, \quad \mathrm{L}(22)=\{2,3\}, \Delta(22)=\{1\}, \\
& \mathrm{Z}(24)=\{(4,0,0),(0,3,0)\}, \quad \mathrm{L}(24)=\{3,4\}, \Delta(24)=\{1\} .
\end{aligned}
$$

Finally, let $S=\langle 6,10,15\rangle$. Then $\operatorname{Betti}(S)=\{30\}$,

$$
\mathrm{Z}(30)=\{(5,0,0),(0,3,0),(0,0,2)\}, \mathrm{L}(30)=\{2,3,5\}, \Delta(30)=\{1,2\} .
$$

More details on the relation between Betti elements and Delta sets can be found in [6].

It is straightforward to see that from the factorizations of every $b \in \operatorname{Betti}(S)$, we can construct $\left\{\mathbf{z}-\mathbf{z}^{\prime} \in M_{S} \mid \mathbf{z} \neq \mathbf{z}^{\prime}, \mathbf{z}, \mathbf{z}^{\prime} \in \mathbf{Z}(b), b \in \operatorname{Betti}(S)\right\}$, which generates $M_{S}$ as a group.

For instance, for $S=\langle 6,8,11\rangle,(1,2,-2)$ and $(4,-3,0)$ generate as a group the set of integer solutions of the equation $6 x_{1}+8 x_{2}+11 x_{3}=0$.

Since the structure of the Delta set of a non-symmetric numerical semigroup is known, we focus on the study of the symmetric numerical semigroups.

\section{Two CASES IN THE SYMMETRIC SETTING}

Let $S$ be a numerical semigroup minimally generated by $\left\{n_{1}, n_{2}, n_{3}\right\}$, and assume that $S$ is symmetric. In this Section, we define the integers and vectors that allow us to study $M_{S}$ and construct $\Delta(S)$.

By Proposition 3, \# Betti $(S) \leq 2$. Now, we going to choose a suitable basis $\left\{\mathbf{v}_{1}, \mathbf{v}_{2}\right\}$ for $M_{S}$ according to \# $\operatorname{Betti}(S)$.

(1) A single Betti element.

If \# Betti $(S)=1$, then $\left(n_{1}, n_{2}, n_{3}\right)=\left(s_{2} s_{3}, s_{1} s_{3}, s_{1} s_{2}\right)$ for some positive pairwise coprime integers $s_{1}>s_{2}>s_{3}$ (see [12]). Moreover, $\operatorname{Betti}(S)=\left\{s_{1} s_{2} s_{3}\right\}$, and the set of factorizations of $s_{1} s_{2} s_{3}$ is $\mathrm{Z}\left(s_{1} s_{2} s_{3}\right)=\left\{\left(s_{1}, 0,0\right),\left(0, s_{2}, 0\right),\left(0,0, s_{3}\right)\right\}$. In this setting, it is easy to see that $M_{S}$ is the group spanned by $\left\{\left(s_{1},-s_{2}, 0\right),\left(0, s_{2},-s_{3}\right)\right\}$. We set $\mathbf{v}_{1}=\left(s_{1},-s_{2}, 0\right), \mathbf{v}_{2}=\left(0, s_{2},-s_{3}\right)$.

(2) Two Betti elements.

If $\# \operatorname{Betti}(S)=2$, we have $\left(n_{1}, n_{2}, n_{3}\right)=\left(a m_{1}, a m_{2}, b m_{1}+c m_{2}\right)$ with $a \geq 2$ and $b+c \geq 2$, and we can also assume that $m_{1}<m_{2}$ [14, Theorem 10.6]. In this setting

$$
\operatorname{Betti}(S)=\left\{a\left(b m_{1}+c m_{2}\right), a m_{1} m_{2}\right\},
$$


with

$$
\begin{aligned}
\mathrm{Z}\left(a\left(b m_{1}+c m_{2}\right)\right)=\{ & \left(b-\left\lfloor\frac{b}{m_{2}}\right\rfloor m_{2}, c+\left\lfloor\frac{b}{m_{2}}\right\rfloor m_{1}, 0\right), \ldots,\left(b-m_{2}, c+m_{1}, 0\right),(b, c, 0), \\
& \left.\left(b+m_{2}, c-m_{1}, 0\right) \ldots,\left(b+\left\lfloor\frac{c}{m_{1}}\right\rfloor m_{2}, c-\left\lfloor\frac{c}{m_{1}}\right\rfloor m_{1}, 0\right),(0,0, a)\right\}
\end{aligned}
$$

and $\mathrm{Z}\left(a m_{1} m_{2}\right)=\left\{\left(m_{2}, 0,0\right),\left(0, m_{1}, 0\right)\right\}$. Moreover, $M_{S}$ is spanned by $\left\{\left(m_{2},-m_{1}, 0\right),(b+\right.$ $\left.\left.\lambda m_{2}, c-\lambda m_{1},-a\right)\right\}$ for any $\lambda \in \mathbb{Z}$. We choose $\lambda \in\left\{-\left\lfloor b / m_{2}\right\rfloor, \ldots,\left\lfloor c / m_{1}\right\rfloor\right\}$ such that $\ell((b+$ $\left.\left.\lambda m_{2}, c-\lambda m_{1},-a\right)\right)$ is minimal. We define $\mathbf{v}_{1}=\left(m_{2},-m_{1}, 0\right)$ and $\mathbf{v}_{2}=\left(b+\lambda m_{2}, c-\lambda m_{1},-a\right)$.

Now, we define $\delta_{1}=\ell\left(\mathbf{v}_{1}\right)$ and $\delta_{2}=\left|\ell\left(\mathbf{v}_{2}\right)\right|$. We consider the absolute value for $\ell\left(\mathbf{v}_{2}\right)$, since it might happen that $\ell\left(\mathbf{v}_{2}\right)<0$ when there are two Betti elements and $a>b+c+\lambda\left(m_{2}-m_{1}\right)$. In order to keep trace of the sign of $\ell\left(\mathbf{v}_{2}\right)$, let $\operatorname{sgn}$ be the sign function, and set $\sigma=\operatorname{sgn}\left(\ell\left(\mathbf{v}_{2}\right)\right)$. In this way, we have $\delta_{2}=\sigma \ell\left(\mathbf{v}_{2}\right)$.

The integers $\delta_{1}, \delta_{2}$ just defined are tightly related to $\Delta(S)$.

Proposition 5. Let $S$ be a symmetric numerical semigroup, and let $\delta_{1}$ and $\delta_{2}$ be defined as above. Then

$$
\max \Delta(S)=\max \left\{\delta_{1}, \delta_{2}\right\}
$$

Proof. We know from [6] that $\max \Delta(S)=\max \{\max \Delta(b) \mid b \in \operatorname{Betti}(S)\}$.

If $\# \operatorname{Betti}(S)=1$, since Z $\left(s_{1} s_{2} s_{3}\right)=\left\{\left(s_{1}, 0,0\right),\left(0, s_{2}, 0\right),\left(0,0, s_{3}\right)\right\}$, we get $\Delta\left(s_{1} s_{2} s_{3}\right)=\left\{s_{1}-\right.$ $\left.s_{2}, s_{2}-s_{3}\right\}=\left\{\delta_{1}, \delta_{2}\right\}$, and the thesis follows.

If $\# \operatorname{Betti}(S)=2$, we need to study $Z(b)$ with $b \in \operatorname{Betti}(S)=\left\{a\left(b m_{1}+c m_{2}\right), a m_{1} m_{2}\right\}$.

- $\mathbf{Z}\left(a m_{1} m_{2}\right)=\left\{\left(m_{2}, 0,0\right),\left(0, m_{1}, 0\right)\right\}$. Hence $\Delta\left(a m_{1} m_{2}\right)=\left\{\delta_{1}\right\}$.

- $\mathrm{Z}\left(a\left(b m_{1}+c m_{2}\right)\right)=\{(0,0, a)\} \cup\left\{\left(b+k m_{2}, c-k m_{1}, 0\right) \mid k \in\left\{-\left\lfloor b / m_{2}\right\rfloor, \ldots,\left\lfloor c / m_{1}\right\rfloor\right\}\right\}$.

Observe that $\mathrm{L}\left(a\left(b m_{1}+c m_{2}\right)\right)=\{a\} \cup\left\{b+c+k\left(m_{2}-m_{1}\right) \mid k \in\left\{-\left\lfloor b / m_{2}\right\rfloor, \ldots,\left\lfloor c / m_{1}\right\rfloor\right\}\right\}$. Consider the following cases.

(1) If $a \leq b+c-\left\lfloor b / m_{2}\right\rfloor\left(m_{2}-m_{1}\right)$, then $\lambda=-\left\lfloor b / m_{2}\right\rfloor$ and $\delta_{2}=b+c-\left\lfloor b / m_{2}\right\rfloor\left(m_{2}-m_{1}\right)-a$. Hence $\Delta\left(a\left(b m_{1}+c m_{2}\right)\right)$ equals $\left\{\delta_{1}, \delta_{2}\right\}$, or $\left\{\delta_{1}\right\}$ if $a=b+c-\left\lfloor b / m_{2}\right\rfloor\left(m_{2}-m_{1}\right)$ (that is, $\left.\delta_{2}=0\right)$.

(2) If $a \geq b+c+\left\lfloor c / m_{1}\right\rfloor\left(m_{2}-m_{1}\right)$, then $\lambda=\left\lfloor c / m_{1}\right\rfloor$, and we argue as in the previous case.

(3) Finally, if $b+c-\left\lfloor b / m_{2}\right\rfloor\left(m_{2}-m_{1}\right)<a<b+c+\left\lfloor c / m_{1}\right\rfloor\left(m_{2}-m_{1}\right)$, there exists $k \in$ $\left\{-\left\lfloor b / m_{2}\right\rfloor, \ldots,\left\lfloor c / m_{1}\right\rfloor-1\right\}$ such that $b+c+k\left(m_{2}-m_{1}\right) \leq a<b+c+(k+1)\left(m_{2}-m_{1}\right)$. Then $\lambda$ is either $k$ or $k+1$, and $\Delta\left(a\left(b m_{1}+c m_{2}\right)\right)=\left\{\delta_{1}, \delta_{2},\left|\delta_{2}-\delta_{1}\right|\right\}$ (unless $\delta_{2}=0$ and $\left.\Delta\left(a\left(b m_{1}+c m_{2}\right)\right)=\left\{\delta_{1}\right\}\right)$.

In any case, $\max \Delta(S)=\max \left\{\delta_{1}, \delta_{2}\right\}$.

The following result is a particular instance of a more general property.

Proposition 6. [6, Corollay 3.1] Let $S$ be a symmetric numerical semigroup. Then

$$
\min \Delta(S)=\operatorname{gcd}\left\{\delta_{1}, \delta_{2}\right\} .
$$

Thus, $\max \Delta(S)$ is either $\delta_{1}$ or $\delta_{2}$, while in our setting $\min \Delta(S)=\operatorname{gcd}\left(\delta_{1}, \delta_{2}\right)$, and each element of $\Delta(S)$ is a multiple of this greatest common divisor [13.

Remark 7 . Observe that, in both cases, the vector $\mathbf{v}_{1}$ has the first coordinate positive, the second one negative, and the third coordinate equal to zero. Our choice of $\lambda$ in the case \#Betti $(S)=2$ ensures that the vector $\mathbf{v}_{2}$ has first coordinate nonnegative, positive the second, and the third coordinate negative. We will represent this fact as:

$$
\mathbf{v}_{1}=(+,-, 0) \text { and } \mathbf{v}_{2}=(+,+,-) .
$$

Proposition 8. Let $S$ be a symmetric numerical semigroup with embedding dimension three. Let $\delta_{1}, \delta_{2}, \mathbf{v}_{1}, \mathbf{v}_{2}$ and $\sigma$ be defined as above.

(1) if $\sigma=1$, we have that $\delta_{2} \mathbf{v}_{1}-\sigma \delta_{1} \mathbf{v}_{2}=(?,-,+)$. 
(2) if $\sigma=-1$, we have that $\delta_{2} \mathbf{v}_{1}-\sigma \delta_{1} \mathbf{v}_{2}=(+, ?,-)$.

The symbol "?" denotes the sign of this coordinate is not determined.

Proof. If $\sigma=1$ and \# $\operatorname{Betti}(S)=1$, we know that $\mathbf{v}_{1}=\left(s_{1},-s_{2}, 0\right)$ and $\mathbf{v}_{2}=\left(0, s_{2},-s_{3}\right)$. Then

$$
\delta_{2} \mathbf{v}_{1}-\delta_{1} \mathbf{v}_{2}=\left(\delta_{2} s_{1},-\delta_{2} s_{2}-\delta_{1} s_{2}, \delta_{1} s_{3}\right)=\left(\delta_{2} s_{1},-s_{2}\left(\delta_{2}+\delta_{1}\right), \delta_{1} s_{3}\right)=(+,-,+) .
$$

For $\sigma=1$ and $\# \operatorname{Betti}(S)=2$, we have $\mathbf{v}_{1}=\left(m_{2},-m_{1}, 0\right)$ and $\mathbf{v}_{2}=\left(b+\lambda m_{2}, c-\lambda m_{1},-a\right)$, whence

$$
\delta_{2} \mathbf{v}_{1}-\delta_{1} \mathbf{v}_{2}=\left(\delta_{2} m_{2}-\delta_{1}\left(b+\lambda m_{2}\right),-\delta_{2} m_{1}-\delta_{1}\left(c-\lambda m_{1}\right), \delta_{1} a\right)=(?,-,+) .
$$

Finally if $\sigma=-1$ and \# $\operatorname{Betti}(S)=2$,

$$
\delta_{2} \mathbf{v}_{1}+\delta_{1} \mathbf{v}_{2}=\left(\delta_{2} m_{2}+\delta_{1}\left(b+\lambda m_{2}\right),-\delta_{2} m_{1}+\delta_{1}\left(c-\lambda m_{1}\right),-\delta_{1} a\right)=(+, ?,-) .
$$

\section{Euclid's Set AND the Delta SET}

Associated to $\delta_{1}$ and $\delta_{2}$ we are going to define its Euclid's set as the set of all integers that appear in the naive implementation of the greatest common divisor algorithm. We will see that precisely this set is the Delta set of the semigroup except to the zero element.

Proposition 9. Let $\delta_{1}, \delta_{2}$ be two positive integers, and let $x \in\left\{1, \ldots, \max \left\{\delta_{1}, \delta_{2}\right\}\right\} \cap g \mathbb{Z}$, where $g=\operatorname{gcd}\left(\delta_{1}, \delta_{2}\right)$. Then there exist unique $\left(x_{1}, x_{2}\right)$ and $\left(x_{1}^{\prime}, x_{2}^{\prime}\right)$ in $\mathbb{Z}^{2}$ such that

(1) $x=x_{1} \delta_{1}+x_{2} \delta_{2}$ with $-\delta_{1} / g<x_{2} \leq 0<x_{1} \leq \delta_{2} / g$,

(2) $x=x_{1}^{\prime} \delta_{1}+x_{2}^{\prime} \delta_{2}$ with $-\delta_{2} / g<x_{1}^{\prime} \leq 0<x_{2}^{\prime} \leq \delta_{1} / g$.

Moreover $\left(x_{1}, x_{2}\right)=\left(x_{1}^{\prime}+\delta_{2} / g, x_{2}^{\prime}-\delta_{1} / g\right)$.

We will denote

- $x^{\left(\delta_{1}, \delta_{2}\right)}=\left(x_{1}, x_{2}\right)$, where $0<x_{1} \leq \delta_{2} / g,-\delta_{1} / g<x_{2} \leq 0$, and $x=x_{1} \delta_{1}+x_{2} \delta_{2}$,

- $x^{\prime\left(\delta_{1}, \delta_{2}\right)}=\left(x_{1}^{\prime}, x_{2}^{\prime}\right)$, where $-\delta_{2} / g<x_{1}^{\prime} \leq 0,0<x_{2} \leq \delta_{1} / g$, and $x=x_{1}^{\prime} \delta_{1}+x_{2}^{\prime} \delta_{2}$.

In particular, $\delta_{1}^{\left(\delta_{1}, \delta_{2}\right)}=(1,0), \delta_{1}^{\prime\left(\delta_{1}, \delta_{2}\right)}=\left(1-\delta_{2} / g, \delta_{1} / g\right), \delta_{2}^{\prime\left(\delta_{1}, \delta_{2}\right)}=(0,1)$ and $\delta_{2}^{\left(\delta_{1}, \delta_{2}\right)}=\left(\delta_{2} / g, 1-\right.$ $\left.\delta_{1} / g\right)$.

We want to depict the set of elements obtained after applying Euclid's greatest common divisor algorithm to $\delta_{1}$ and $\delta_{2}$. We will use the naive approach that uses substraction instead of remainders of divisions. Our set will be decomposed in subsets corresponding to the algorithm using remainders.

Definition 10. Let $\delta_{1}$ and $\delta_{2}$ be positive integers, and define $\eta_{1}=\max \left\{\delta_{1}, \delta_{2}\right\}, \eta_{2}=\min \left\{\delta_{1}, \delta_{2}\right\}$ and $\eta_{3}=\eta_{1} \bmod \eta_{2}$. In general, for $j>0$, define $\eta_{j+2}=\eta_{j}-\left\lfloor\frac{\eta_{j}}{\eta_{j+1}}\right\rfloor \eta_{j+1}=\eta_{j} \bmod \eta_{j+1}$. Let $i$ be the maximum index such that $\eta_{i+1}>0$. Define

$$
\begin{aligned}
& \mathrm{D}\left(\eta_{1}, \eta_{2}\right)=\left\{\eta_{1}, \eta_{1}-\eta_{2}, \ldots, \eta_{1} \bmod \eta_{2}=\eta_{3}\right\}, \\
& \mathrm{D}\left(\eta_{2}, \eta_{3}\right)=\left\{\eta_{2}, \eta_{2}-\eta_{3}, \ldots, \eta_{2} \bmod \eta_{3}=\eta_{4}\right\}, \\
& \mathrm{D}\left(\eta_{3}, \eta_{4}\right)=\left\{\eta_{3}-\eta_{4}, \ldots, \eta_{3} \bmod \eta_{4}=\eta_{5}\right\}, \\
& \cdots \\
& \mathrm{D}\left(\eta_{j-1}, \eta_{j}\right)=\left\{\eta_{j-1}-\eta_{j}, \ldots, \eta_{j-1} \bmod \eta_{j}=\eta_{j+1}\right\}, \\
& \cdots \\
& \mathrm{D}\left(\eta_{i}, \eta_{i+1}\right)=\left\{\eta_{i}-\eta_{i+1}, \ldots, \eta_{i} \bmod \eta_{i+1}=\eta_{i+2}=0\right\}
\end{aligned}
$$

Let us denote by $\operatorname{Euc}\left(\delta_{1}, \delta_{2}\right)=\bigcup_{i \in I} \mathrm{D}\left(\eta_{i}, \eta_{i+1}\right)$, where $I=\left\{i \in \mathbb{N} \mid \eta_{i+1}>0\right\}$.

Observe that $\eta_{1} \in \mathrm{D}\left(\eta_{1}, \eta_{2}\right)$ and $\eta_{2} \in \mathrm{D}\left(\eta_{2}, \eta_{3}\right)$, but this is no longer the case for $i>2$. This is because we want the union in the definition of $\operatorname{Euc}\left(\delta_{1}, \delta_{2}\right)$ to be disjoint. Also, $\eta_{j} \in \mathrm{D}\left(\eta_{j-2}, \eta_{j-1}\right)$ as $\eta_{j}=\eta_{j-2}-\left\lfloor\eta_{j-2} / \eta_{j-1}\right\rfloor \eta_{j-1}$ for any $j>2$.

Notice also that $\operatorname{Euc}\left(\delta_{1}, \delta_{2}\right) \subset g \mathbb{Z}$ where $g=\operatorname{gcd}\left(\delta_{1}, \delta_{2}\right)$.

Example 11. Let $S=\left\langle s_{2} s_{3}, s_{1} s_{3}, s_{1} s_{2}\right\rangle$, with $s_{1}=548, s_{2}=155$, and $s_{3}=13$. Then $\mathbf{v}_{1}=$ $(548,-155,0)$ and $\mathbf{v}_{2}=(0,155,-13)$. Hence $\delta_{1}=393$ and $\delta_{2}=142$. In this setting, 
- $\mathrm{D}(393,142)=\{393,251,109\}$,

- $\mathrm{D}(142,109)=\{142,33\}$,

- $\mathrm{D}(109,33)=\{76,43,10\}$,

- $\mathrm{D}(33,10)=\{23,13,3\}$,

- $\mathrm{D}(10,3)=\{7,4,1\}$,

- $\mathrm{D}(3,1)=\{2,1,0\}$.

Then $\operatorname{Euc}\left(\delta_{1}, \delta_{2}\right)=\{0,1,2,3,4,7,10,13,23,33,43,76,109,142,251,393\}$.

Remark 12. It is clear that $\eta_{1}>\eta_{2}>\cdots>\eta_{i}>\eta_{i+1}>\eta_{i+2}=0$, and for $\eta_{i}^{\left(\eta_{1}, \eta_{2}\right)}=\left(\eta_{i 1}, \eta_{i 2}\right)$ and $\eta_{i+1}^{\prime\left(\eta_{1}, \eta_{2}\right)}=\left(\eta_{i+11}^{\prime}, \eta_{i+12}^{\prime}\right)$, the inequalities $\eta_{i+11}^{\prime} \eta_{i 1} \leq 0$ and $\eta_{i+12}^{\prime} \eta_{i 2} \leq 0$ hold. From these, we have too that, for $q>0,\left|\eta_{i 1}\right| \leq\left|\eta_{i 1}-q \eta_{i+11}^{\prime}\right|$ and $\left|\eta_{i 2}\right| \leq\left|\eta_{i 2}-q \eta_{i+12}^{\prime}\right|$ hold. Analogously, considering $\eta_{i}^{\prime\left(\eta_{1}, \eta_{2}\right)}=\left(\eta_{i 1}^{\prime}, \eta_{i 2}^{\prime}\right)$ and $\eta_{i+1}^{\left(\eta_{1}, \eta_{2}\right)}=\left(\eta_{i+11}, \eta_{i+12}\right)$, we have $\left|\eta_{i 1}^{\prime}\right| \leq\left|\eta_{i 1}^{\prime}-q \eta_{i+11}\right|,\left|\eta_{i 2}^{\prime}\right| \leq\left|\eta_{i 2}^{\prime}-q \eta_{i+12}\right|$ for $q>0$. Observe that we have an equality only when one of the $\eta_{h k}$ is zero.

As $\eta_{1}^{\left(\eta_{1}, \eta_{2}\right)}=(1,0)$ and $\eta_{2}^{\prime\left(\eta_{1}, \eta_{2}\right)}=(0,1)=(-,+)$, we obtain $\eta_{3}^{\left(\eta_{1}, \eta_{2}\right)}=\left(1,-\left\lfloor\eta_{1} / \eta_{2}\right\rfloor\right)=(+,-)$ and $\eta_{4}^{\prime\left(\eta_{1}, \eta_{2}\right)}=\left(-\left\lfloor\eta_{2} / \eta_{3}\right\rfloor, 1+\left\lfloor\eta_{2} / \eta_{3}\right\rfloor\left\lfloor\eta_{1} / \eta_{2}\right\rfloor\right)=(-,+)$, and so on.

This means that in each step in $\operatorname{Euc}\left(\delta_{1}, \delta_{2}\right)$, the absolute value of the $\left(\eta_{1}, \eta_{2}\right)$-coordinates increases, (and is not decreasing only when $x_{h k}=x_{21}^{\prime}$ ). We will use this fact in the next propositions.

Proposition 13. Let the notation and hypotheses be as in Definition [10, and let $d \in\left\{\eta_{3}, \eta_{3}+\right.$ $\left.g, \ldots, \eta_{1}\right\}$. Then $d \in \mathrm{D}\left(\eta_{1}, \eta_{2}\right)$ if and only if $d_{1}=1$, with $\left(d_{1}, d_{2}\right)=d^{\left(\eta_{1}, \eta_{2}\right)}$ (according to the notation given in Proposition (9).

Proof. Let $d \in\left\{\eta_{3}, \eta_{3}+g, \ldots, \eta_{1}\right\}$. If $d \in \mathrm{D}\left(\eta_{1}, \eta_{2}\right)$, then $d=\eta_{1}-k \eta_{2}$ for some $k \in\left\{0, \ldots,\left\lfloor\eta_{1} / \eta_{2}\right\rfloor\right\}$. Hence $d^{\left(\eta_{1}, \eta_{2}\right)}=(1,-k)$. For the converse, if $0<d=\eta_{1}+k \eta_{2} \leq \eta_{1}$ for some $k \in \mathbb{Z}$, we obtain $-\eta_{1}<k \eta_{2} \leq 0$, whence $-\eta_{1} / \eta_{2}<k \leq 0$. Now, as $g=\operatorname{gcd}\left(\delta_{1}, \delta_{2}\right) \leq \eta_{2}$ we have $-\eta_{1} / g \leq-\eta_{1} / \eta_{2}<k$ and by Proposition 9, $k$ will be equal to $d_{2}$, and consequently $d \in \mathrm{D}\left(\eta_{1}, \eta_{2}\right)$.

Proposition 14. Let the notation and hypotheses be as in Definition 10.

(1) Let $x \in\left\{\eta_{3}, \eta_{3}+g, \ldots, \eta_{1}\right\} \backslash\left(\mathrm{D}\left(\eta_{1}, \eta_{2}\right) \cup \mathrm{D}\left(\eta_{2}, \eta_{3}\right)\right)$.

(a) If $x^{\left(\eta_{1}, \eta_{2}\right)}=\left(x_{1}, x_{2}\right)=(+,-)$, then there exists an integer $d<x, d \in \mathrm{D}\left(\eta_{1}, \eta_{2}\right)$, such that $d^{\left(\eta_{1}, \eta_{2}\right)}=\left(d_{1}, d_{2}\right)$ with $0<d_{1}<x_{1}$ and $x_{2}<d_{2}<0$.

(b) If $x^{\prime\left(\eta_{1}, \eta_{2}\right)}=\left(x_{1}^{\prime}, x_{2}^{\prime}\right)=(-,+)$, then there exists an integer $d<x, d \in \mathrm{D}\left(\eta_{2}, \eta_{3}\right)$, such that $d^{\prime\left(\eta_{1}, \eta_{2}\right)}=\left(d_{1}^{\prime}, d_{2}^{\prime}\right)$ with $x_{1}^{\prime} \leq d_{1}^{\prime} \leq 0$ and $0<d_{2}^{\prime}<x_{2}^{\prime}$.

The case $x_{1}^{\prime}=d_{1}^{\prime}=0$ corresponds to $x=d_{2} \eta_{2}$, that is, only when $x$ is a multiple of $\eta_{2}$.

(2) In general, for any $x$ multiple of $g$ such that $x<\eta_{3}, x \notin \operatorname{Euc}\left(\delta_{1}, \delta_{2}\right)$, consider $j+1=\min \{k \in$ $\left.I \mid \eta_{k}<x\right\}$ and then $x \in\left\{\eta_{j+1}, \eta_{j+1}+g, \ldots, \eta_{j}\right\} \backslash\left(\mathrm{D}\left(\eta_{j-1}, \eta_{j}\right) \cup \mathrm{D}\left(\eta_{j}, \eta_{j+1}\right)\right)=\left\{\eta_{j+1}, \eta_{j+1}+\right.$ $\left.g, \ldots, \eta_{j}\right\} \backslash \operatorname{Euc}\left(\delta_{1}, \delta_{2}\right)$.

(a) If $x^{\left(\eta_{1}, \eta_{2}\right)}=\left(x_{1}, x_{2}\right)=(+,-)$, then there exists an integer $d<x, d \in \mathrm{D}\left(\eta_{j-1}, \eta_{j}\right) \cup$ $\mathrm{D}\left(\eta_{j}, \eta_{j+1}\right)$, such that $d^{\left(\eta_{1}, \eta_{2}\right)}=\left(d_{1}, d_{2}\right)$ with $0<d_{1}<x_{1}$ and $x_{2}<d_{2}<0$.

(b) If $x^{\prime\left(\eta_{1}, \eta_{2}\right)}=\left(x_{1}^{\prime}, x_{2}^{\prime}\right)=(-,+)$, then there exists an integer $d<x, d \in \mathrm{D}\left(\eta_{j-1}, \eta_{j}\right) \cup$ $\mathrm{D}\left(\eta_{j}, \eta_{j+1}\right)$, such that $d^{\prime\left(\eta_{1}, \eta_{2}\right)}=\left(d_{1}^{\prime}, d_{2}^{\prime}\right)$ with $x_{1}^{\prime}<d_{1}^{\prime}<0$ and $0<d_{2}^{\prime}<x_{2}^{\prime}$.

Proof. (1) We study each case independently.

(a) As $\eta_{1}-\left\lfloor\frac{\eta_{1}}{\eta_{2}}\right\rfloor \eta_{2}=\eta_{3}<x<\eta_{1}$ and $x \notin \mathrm{D}\left(\eta_{1}, \eta_{2}\right) \cup \mathrm{D}\left(\eta_{2}, \eta_{3}\right)$, there exists an integer $k$ such that $\eta_{1}+k \eta_{2}<x<\eta_{1}+(k+1) \eta_{2}$ and $-\left\lfloor\frac{\eta_{1}}{\eta_{2}}\right\rfloor<k<0$. Set $d=\eta_{1}+k \eta_{2}$. Then $d \in \mathrm{D}\left(\eta_{1}, \eta_{2}\right)$. Also, $\eta_{2} \geq g$ and $\delta_{1} \leq \eta_{1}$, thus $-\frac{\delta_{1}}{g} \leq-\frac{\eta_{1}}{\eta_{2}} \leq\left\lceil-\frac{\eta_{1}}{\eta_{2}}\right\rceil=-\left\lfloor\frac{\eta_{1}}{\eta_{2}}\right\rfloor<k$. This implies that $d^{\left(\eta_{1}, \eta_{2}\right)}=(1, k)$. It is clear that $x_{1} \geq 2$ by Proposition [13, and as $d_{1}=1$ we have that $0<d_{1}<x_{1}$. Also, as $x=x_{1} \eta_{1}+x_{2} \eta_{2}$ and $x_{1} \geq 2$, and we obtain that $2 \eta_{1}+x_{2} \eta_{2} \leq x<\eta_{1}+(k+1) \eta_{2}$. Consequently, $x_{2} \eta_{2}<\eta_{1}+x_{2} \eta_{2}<(k+1) \eta_{2}$. Thus, $x_{2}<k+1$. Observe that $x_{2} \neq k$, since otherwise $x \geq 2 \eta_{1}+k \eta_{2}>\eta_{1}+\eta_{2}+k \eta_{2}=\eta_{1}+(k+1) \eta_{2}$, a contradiction. Hence $x_{2}<k=d_{2}<0$. 
(b) For $\left(x_{1}^{\prime}, x_{2}^{\prime}\right)$ we distinguish two cases.

(i) If $x>\eta_{2}$, it suffices to take $d=\eta_{2} \in \mathrm{D}\left(\eta_{2}, \eta_{3}\right)$. We have that $d_{1}^{\prime}=0$ and $d_{2}^{\prime}=1$. In light of Proposition 9, it follows that $x_{1}^{\prime} \leq d_{1}^{\prime}=0$ and $0<d_{2}^{\prime}<x_{2}^{\prime}$. It is clear that $x_{2}^{\prime} \neq 1$, since otherwise we would have $x=x_{1}^{\prime} \eta_{1}+x_{2}^{\prime} \eta_{2}=x_{1}^{\prime} \eta_{1}+\eta_{2} \leq d_{1}^{\prime} \eta_{1}+\eta_{2}=\eta_{2}$, that is, $x \leq \eta_{2}$, a contradiction.

(ii) If $\eta_{3}<x<\eta_{2}$, consider $\mathrm{D}\left(\eta_{2}, \eta_{3}\right)=\left\{\eta_{2}, \eta_{2}-\eta_{3}, \eta_{2}-2 \eta_{3}, \ldots, \eta_{4}\right\}$. As $x \notin \mathrm{D}\left(\eta_{2}, \eta_{3}\right)$, by denoting $x^{\left(\eta_{2}, \eta_{3}\right)}=\left(x_{2}, x_{3}\right)=(+,-)$ we can, as above, choose and integer $h$ such that $\eta_{2}+h \eta_{3}<x<\eta_{2}+(h+1) \eta_{3}$. Then, taking $d=\eta_{2}+h \eta_{3}$ and arguing as in (a) with $d=\eta_{2}+h \eta_{3}$ and $x=x_{2} \eta_{2}+x_{3} \eta_{3}$, we have that $0<1<x_{2}$ and $x_{3}<h<0$. Now, as $\eta_{3}=\eta_{1} \bmod \eta_{2}=\eta_{1}-\left\lfloor\eta_{1} / \eta_{2}\right\rfloor \eta_{2}$, we can rewrite

$$
\begin{aligned}
& x=x_{2} \eta_{2}+x_{3}\left(\eta_{1}-\left\lfloor\eta_{1} / \eta_{2}\right\rfloor \eta_{2}\right)=x_{3} \eta_{1}+\left(x_{2}-x_{3}\left\lfloor\eta_{1} / \eta_{2}\right\rfloor\right) \eta_{2}, \\
& d=\eta_{2}+h\left(\eta_{1}-\left\lfloor\eta_{1} / \eta_{2}\right\rfloor \eta_{2}\right)=h \eta_{1}+\left(1-h\left\lfloor\eta_{1} / \eta_{2}\right\rfloor\right) \eta_{2} .
\end{aligned}
$$

Thus, $\left(x_{1}^{\prime}, x_{2}^{\prime}\right)^{\left(\eta_{1}, \eta_{2}\right)}=\left(x_{3}, x_{2}-x_{3}\left\lfloor\eta_{1} / \eta_{2}\right\rfloor\right)$ and $\left(d_{1}^{\prime}, d_{2}^{\prime}\right)^{\left(\eta_{1}, \eta_{2}\right)}=\left(h, 1-h\left\lfloor\eta_{1} / \eta_{2}\right\rfloor\right)$. Moreover, it is clear that $x_{1}^{\prime}=x_{3}<h=d_{1}^{\prime}<0$. We also have that $-x_{3}\left\lfloor\eta_{1} / \eta_{2}\right\rfloor>$ $-h\left\lfloor\eta_{1} / \eta_{2}\right\rfloor>0$ and $x_{2}>1>0$. Hence $x_{2}^{\prime}=x_{2}-x_{3}\left\lfloor\eta_{1} / \eta_{2}\right\rfloor>1-h\left\lfloor\eta_{1} / \eta_{2}\right\rfloor=d_{2}^{\prime}>0$.

(2) For the general case, we will follow the arguments of the preceding cases. It is clear that for all suitable $j, \operatorname{gcd}\left(\eta_{j}, \eta_{j+1}\right)=\operatorname{gcd}\left(\delta_{1}, \delta_{2}\right)=g$. Hence we have the following.

- Observe that $j \in I$ implies $\eta_{j+1}>0$, so an element $d \in\left\{\eta_{j+1}, \eta_{j+1}+g, \ldots, \eta_{j}\right\}$ is in $\mathrm{D}\left(\eta_{j-1}, \eta_{j}\right) \backslash\left\{\eta_{j}\right\}$ if and only if $d_{1}^{(j-1)}=1$, where $d^{\left(\eta_{j-1}, \eta_{j}\right)}=\left(d_{1}^{(j-1)}, d_{2}^{(j-1)}\right)$. This is the same argument used in Proposition [13, applied for $\eta_{1}=\eta_{j-1}$ and for $\eta_{2}=\eta_{j}$.

- Working at this $(j-1, j)$ level, it is also clear that, if $x \in\left\{\eta_{j+1}, \eta_{j+1}+g, \ldots, \eta_{j}\right\} \backslash\left(\mathrm{D}\left(\eta_{j-1}, \eta_{j}\right) \cup\right.$ $\left.\mathrm{D}\left(\eta_{j}, \eta_{j+1}\right)\right)$, then

(a) if $x^{\left(\eta_{j-1}, \eta_{j}\right)}=\left(x_{1}^{(j-1)}, x_{2}^{(j-1)}\right)=(+,-)$, then there exists $d<x, d \in \mathrm{D}\left(\eta_{j-1}, \eta_{j}\right)$, such that $d^{\left(\eta_{j-1}, \eta_{j}\right)}=\left(d_{1}^{(j-1)}, d_{2}^{(j-1)}\right)$ with $0<d_{1}^{(j-1)}<x_{1}^{(j-1)}$ and $x_{2}^{(j-1)}<d_{2}^{(j-1)}<0$; and

(b) if $x^{\prime\left(\eta_{j-1}, \eta_{j}\right)}=\left(x_{1}^{\prime(j-1)}, x_{2}^{\prime(j-1)}\right)=(-,+)$, then there exists $d<x, d \in \mathrm{D}\left(\eta_{j}, \eta_{j+1}\right)$, such that $d^{\prime\left(\eta_{j-1}, \eta_{j}\right)}=\left(d_{1}^{\prime(j-1)}, d_{2}^{\prime(j-1)}\right)$ with $x_{1}^{\prime(j-1)} \leq d_{1}^{\prime(j-1)} \leq 0$ and $0<d_{2}^{\prime(j-1)}<d_{2}^{\prime(j-1)}$.

We only need to apply the preceding cases at this $(j-1, j)$ level.

It remains to prove that the inequalities for the $(j-1, j)$ level (denoted by the bracketed superscript) can be translated to the first level. To this end, we proceed as in the previous point.

(a) Assume that $0 \leq d_{1}^{(j-1)}<x_{1}^{(j-1)}$ and $x_{2}^{(j-1)}<d_{2}^{(j-1)}<0$. Notice that $d_{1}^{(j-1)}=0$ if and only if $d=\eta_{j}$. As $x=x_{1}^{(j-1)} \eta_{j-1}+x_{2}^{(j-1)} \eta_{j}, d=d_{1}^{(j-1)} \eta_{j-1}+d_{2}^{(j-1)} \eta_{j}$ and $\eta_{j}=$ $\eta_{j-2}-\left\lfloor\frac{\eta_{j-2}}{\eta_{j-1}}\right\rfloor \eta_{j-1}$, we have that

$$
\begin{aligned}
x & =x_{1}^{(j-1)} \eta_{j-1}+x_{2}^{(j-1)}\left(\eta_{j-2}-\left\lfloor\frac{\eta_{j-2}}{\eta_{j-1}}\right\rfloor \eta_{j-1}\right) \\
& =x_{2}^{(j-1)} \eta_{j-2}+\left(x_{1}^{(j-1)}-x_{2}^{(j-1)}\left\lfloor\frac{\eta_{j-2}}{\eta_{j-1}}\right\rfloor\right) \eta_{j-1}, \\
d & =d_{1}^{(j-1)} \eta_{j-1}+d_{2}^{(j-1)}\left(\eta_{j-2}-\left\lfloor\frac{\eta_{j-2}}{\eta_{j-1}}\right\rfloor \eta_{j-1}\right) \\
& =d_{2}^{(j-1)} \eta_{j-2}+\left(d_{1}^{(j-1)}-d_{2}^{(j-1)}\left\lfloor\frac{\eta_{j-2}}{\eta_{j-1}}\right\rfloor\right) \eta_{j-1} .
\end{aligned}
$$

From these equalities we can deduce that $x_{1}^{(j-2)}=x_{2}^{(j-1)}<d_{2}^{(j-1)}=d_{1}^{(j-2)}<0$ and $x_{2}^{\prime(j-2)}=x_{1}^{(j-1)}-x_{2}^{(j-1)}\left\lfloor\frac{\eta_{j-2}}{\eta_{j-1}}\right\rfloor>d_{1}^{(j-1)}-d_{2}^{(j-1)}\left\lfloor\frac{\eta_{j-2}}{\eta_{j-1}}\right\rfloor=d_{2}^{(j-2)}>0$, where $x^{\left(\eta_{j-2}, \eta_{j-1}\right)}=$ $\left(x_{1}^{\prime(j-2)}, x_{2}^{\prime(j-2)}\right)=(-,+)$, and $d^{\prime\left(\eta_{j-2}, \eta_{j-1}\right)}=\left(d_{1}^{\prime(j-2)}, d_{2}^{\prime(j-2)}\right)=(-,+)$. Notice that we can ensure that in this setting all the inequalities are strict. 
(b) Assume that $0 \geq d_{1}^{(j-1)} \geq x_{1}^{\prime(j-1)}$ and $x_{2}^{(j-1)}>d_{2}^{(j-1)}>0$. Since $x=x_{1}^{(j-1)} \eta_{j-1}+$ $x_{2}^{\prime(j-1)} \eta_{j}, d=d_{1}^{(j-1)} \eta_{j-1}+d_{2}^{\prime(j-1)} \eta_{j}$ and $\eta_{j}=\eta_{j-2}-\left\lfloor\frac{\eta_{j-2}}{\eta_{j-1}}\right\rfloor \eta_{j-1}$ we have

$$
\begin{aligned}
x & =x_{1}^{\prime(j-1)} \eta_{j-1}+x_{2}^{\prime(j-1)}\left(\eta_{j-2}-\left\lfloor\frac{\eta_{j-2}}{\eta_{j-1}}\right\rfloor \eta_{j-1}\right) \\
& =x_{2}^{\prime(j-1)} \eta_{j-2}+\left(x_{1}^{\prime(j-1)}-x_{2}^{\prime(j-1)}\left\lfloor\frac{\eta_{j-2}}{\eta_{j-1}}\right\rfloor\right) \eta_{j-1}, \\
d & =d_{1}^{\prime(j-1)} \eta_{j-1}+d_{2}^{\prime(j-1)}\left(\eta_{j-2}-\left\lfloor\frac{\eta_{j-2}}{\eta_{j-1}}\right\rfloor \eta_{j-1}\right) \\
& =d_{2}^{\prime(j-1)} \eta_{j-2}+\left(d_{1}^{\prime(j-1)}-d_{2}^{\prime(j-1)}\left\lfloor\frac{\eta_{j-2}}{\eta_{j-1}}\right\rfloor\right) \eta_{j-1} .
\end{aligned}
$$

Again, it follows that $x_{1}^{(j-2)}=x_{2}^{\prime(j-1)}>d_{2}^{(j-1)}=d_{1}^{(j-2)}>0$ and $x_{2}^{(j-2)}=x_{1}^{\prime(j-1)}-$ $x_{2}^{\prime(j-1)}\left\lfloor\frac{\eta_{j-2}}{\eta_{j-1}}\right\rfloor<d_{1}^{(j-1)}-d_{2}^{\prime(j-1)}\left\lfloor\frac{\eta_{j-2}}{\eta_{j-1}}\right\rfloor=d_{2}^{(j-2)} \leq 0$.

Notice that the inequality is strict because $\left\lfloor\frac{\eta_{j-2}}{\eta_{j-1}}\right\rfloor \geq 1$ and we have $x_{2}^{\prime(j-1)}<d_{2}^{(j-1)}$. Also $d_{1}^{(j-1)}=0$ implies $d=d_{2}^{\prime(j-1)} \eta_{j-2}$, but since $d \in \operatorname{Euc}\left(\delta_{1}, \delta_{2}\right)$, we deduce $d_{2}^{(j-1)}=1$, that is, we always can take $d=\eta_{j+1}$ in the $(-,+)$ case to start the process.

In this way, we can apply alternatively the preceding processes to obtain the desired result. Notice that only if we start with (b), we can have $d_{1}^{\prime j-1}=0$. Hence, only when $j-1=1$ (if we perform another step, when applying (a) the inequality becomes strict), we will have that the $\left(\eta_{1}, \eta_{2}\right)$-coordinates of $d$ would be $(0,1)$, that is, $d=\eta_{2}$.

However, as the statement of (3) assumes $j>2$, the inequalities will always be strict.

We will refer to $d$ defined in Proposition 14 as the basement of $x=\left(x_{1}, x_{2}\right)$, denoted by $\operatorname{bsm}(x)$. Notice that the basement of $x$ may not be unique: however, for our purposes, we will consider as the basement of $x$ any $d$ satisfying the properties stated in Proposition 14 .

Example 15. In the setting of Example 11, consider $x=35$. Since $33=\eta_{4} \leq x \leq 109=\eta_{3}$, according to Proposition 14 we can take $j=3$. As $35=-13 \eta_{3}+44 \eta_{4}=20 \eta_{3}-65 \eta_{4}$; we have $x^{\left(\eta_{3}, \eta_{4}\right)}=(20,-65)$ and $x^{\prime\left(\eta_{3}, \eta_{4}\right)}=(-13,44)$. Remember that these decompositions are unique using Proposition 9 ,

For $x^{\prime\left(\eta_{3}, \eta_{4}\right)}=(-13,44)$ we know that $d^{\prime}=\eta_{4}=33$ and $d^{\prime\left(\eta_{3}, \eta_{4}\right)}=(0,1)$; while we look for $d \in \mathrm{D}\left(\eta_{3}, \eta_{4}\right)=\{76,43,10\}$. As $10<x=35<43$ we take $d=10$ and then $d^{\left(\eta_{3}, \eta_{4}\right)}=(1,-3)$. If we translate them to level $\left(\delta_{1}, \delta_{2}\right)$, we have: $35=-57 \delta_{1}+158 \delta_{2}=85 \delta_{1}-235 \delta_{2}$, and $\left(x_{1}, x_{2}\right)=$ $(85,-235)$ and $\left(x_{1}^{\prime} x_{2}^{\prime}\right)=(-57,158)$. Thus, considering $d^{\prime}=\eta_{4}=33$, as $33=-\eta_{1}+3 \eta_{2}$, we have $d^{\prime\left(\delta_{1}, \delta_{2}\right)}=\left(d_{1}^{\prime}, d_{2}^{\prime}\right)=(-1,3)$. For the other case, by considering $d=10$, we obtain $10=4 \eta_{1}-11 \eta_{2}$, that is, $d^{\left(\delta_{1}, \delta_{2}\right)}=\left(d_{1}, d_{2}\right)=(4,-11)$. So we have $x_{1}^{\prime}<d_{1}^{\prime}<0<d_{2}^{\prime}<x_{2}^{\prime}$ and $x_{2}<d_{2}<0<d_{1}<x_{1}$.

Observe that 33 is the $d^{\prime}$ that yields Proposition 14, but it may happen that there are other elements in $\operatorname{Euc}\left(\delta_{1}, \delta_{2}\right)$ satisfying our purposes too.

It is not difficult to see that for any element in $\{34, \ldots, 42\}$ we obtain the same results, that is, $d=10$ and $d^{\prime}=33$, while if we take a number comprised between 44 and 75 , we will obtain $d=43$ and $d^{\prime}=33$.

Let us, now, consider $x=15$ which is between $\eta_{5}=10$ and $\eta_{4}=33$. In this case $j=4$, then we can write $15=-5 \eta_{4}+18 \eta_{5}=5 \eta_{4}-15 \eta_{5}$. So, for $x^{\prime\left(\eta_{4}, \eta_{5}\right)}=(-5,18)$ we know that $d^{\prime}=\eta_{5}=10$ and $d^{\prime\left(\eta_{4}, \eta_{5}\right)}=(0,1)$; while as $d \in \mathrm{D}\left(\eta_{4}, \eta_{5}\right)=\{23,13,3\}$, we can take $d=13<x=15$ which yields $d^{\left(\eta_{4}, \eta_{5}\right)}=(1,-2)$, satisfying Proposition 14 as $x^{\left(\eta_{4}, \eta_{5}\right)}=(5,-15)$. Observe that, in this case, when we compute the basements at the first level, we obtain $d=10$ because $d^{\left(\delta_{1}, \delta_{2}\right)}=(4,-11)$, while $x^{\left(\delta_{1}, \delta_{2}\right)}=(77,-213)$, and $d^{\prime}=33$ with $d^{\prime\left(\delta_{1}, \delta_{2}\right)}=\left(d_{1}^{\prime}, d_{2}^{\prime}\right)=(-1,3)$ for $x^{\prime\left(\delta_{1}, \delta_{2}\right)}=(-65,180)$, changing the roles of $d$ and $d^{\prime}$ from level $j=4$ to the first level. 
Our next goal is to associate $\operatorname{bsm}(x)$ to a vector in $M_{S}$. We will have two such vectors per case, associated to the decompositions in Proposition 9 .

As above $g=\operatorname{gcd}\left(\delta_{1}, \delta_{2}\right)$. For $x \in\left\{g, 2 g, \ldots, \max \left\{\delta_{1}, \delta_{2}\right\}\right\}$, and $\left\{\mathbf{v}_{1}, \mathbf{v}_{2}\right\}$ the basis for $M_{S}$ chosen in Section 3, set

$$
\mathbf{w}_{x}=x_{1} \mathbf{v}_{1}+\sigma x_{2} \mathbf{v}_{2}, \quad \mathbf{w}_{x}^{\prime}=x_{1}^{\prime} \mathbf{v}_{1}+\sigma x_{2}^{\prime} \mathbf{v}_{2},
$$

with $\left(x_{1}, x_{2}\right)=x^{\left(\delta_{1}, \delta_{2}\right)}$ and $\left(x_{1}^{\prime}, x_{2}^{\prime}\right)=x^{\prime\left(\delta_{1}, \delta_{2}\right)}$. Notice that in this setting $\ell\left(\mathbf{w}_{x}\right)=x_{1} \delta_{1}+x_{2} \delta_{2}=$ $x=x_{1}^{\prime} \delta_{1}+x_{2}^{\prime} \delta_{2}=\ell\left(\mathbf{w}_{x}^{\prime}\right)$.

In the following, we will use the bracketed subscript to refer to the coordinates of vectors. For instance, we would have, for a generic vector $\mathbf{v}, \mathbf{v}=\left(v_{(1)}, v_{(2)}, v_{(3)}\right)$. In particular, we will use the following notation to refer to the coordinates of the vectors $\mathbf{w}_{x}$ and $\mathbf{w}_{x}^{\prime}$ we just introduced:

$$
\mathbf{w}_{x}=\left(w_{x_{(1)}}, w_{x_{(2)}}, w_{x_{(3)}}\right) \text { and } \mathbf{w}_{x}^{\prime}=\left(w_{x_{(1)}}^{\prime}, w_{x_{(2)}}^{\prime}, w_{x_{(3)}}^{\prime}\right) .
$$

As $x_{1}>0$ and $x_{2} \leq 0$, following an argument similar to the proof of Proposition 8 , it can be shown that the signs of the coordinates of $\mathbf{w}_{x}$ and $\mathbf{w}_{x}^{\prime}$ are as in Table 1.

\begin{tabular}{c|cc}
$\sigma$ & $\mathbf{w}_{x}$ & $\mathbf{w}_{x}^{\prime}$ \\
\hline 1 & $(?,-,+)$ & $(?,+,-)$ \\
-1 & $(+, ?,-)$ & $(-, ?,+)$
\end{tabular}

TABLE 1. Signs for $\mathbf{w}_{x}$ and $\mathbf{w}_{x}^{\prime}$

Remember that $\mathbf{v}_{1}=(+,-, 0)$ and $\mathbf{v}_{2}=(+,+,-)$.

The following corollary shows that, for $x$ as in Proposition 14, the vector $\operatorname{bsm}(x)$ has two coordinates that are in absolute value lower than those of $\mathbf{w}_{x}$ or $\mathbf{w}_{x}^{\prime}$.

Corollary 16. Let the notations and hypotheses be as above. Let $\mathbf{v}=a_{1} \mathbf{v}_{1}+a_{2} \sigma \mathbf{v}_{2}$, with $a_{1}$ and $a_{2}$ integers such that $a_{1} a_{2} \leq 0,-\delta_{2} / g<a_{1} \leq \delta_{2} / g$ and $-\delta_{1} / g<a_{2} \leq \delta_{1} / g$. Set $x=\ell(\mathbf{v}) \in$ $\left\{g, 2 g, \ldots, \max \left\{\delta_{1}, \delta_{2}\right\}\right\} \backslash \operatorname{Euc}\left(\delta_{1}, \delta_{2}\right)$, and let $d=\operatorname{bsm}(x)$.

(i) If $a_{1}>0$ (that is, $\mathbf{v}=\mathbf{w}_{x}$ ), there exists $i \in\{1,2\}$ such that $\left|w_{d_{(i)}}\right|<\left|w_{x_{(i)}}\right|$ and $\left|w_{d_{(3)}}\right|<$ $\left|w_{x_{(3)}}\right|$. Moreover, for such an $i$ we have that $w_{d_{(i)}} w_{d_{(3)}}<0$.

(ii) If $a_{1}<0$ (that is, $\mathbf{v}=\mathbf{w}_{x}^{\prime}$ ), there exists $i \in\{1,2\}$ such that $\left|w_{d_{(i)}}^{\prime}\right|<\left|w_{x_{(i)}}^{\prime}\right|$ and $\left|w_{d_{(3)}}^{\prime}\right|<$ $\left|w_{x_{(3)}}^{\prime}\right|$. Moreover, for such an $i$ we have that $w_{d_{(i)}}^{\prime} w_{d_{(3)}}^{\prime}<0$.

Proof. Notice that $x=a_{1} \delta_{1}+a_{2} \delta_{2}$. According to Proposition 9 , if $a_{1}>0$, then $\left(a_{1}, a_{2}\right)=x^{\left(\delta_{1}, \delta_{2}\right)}$; otherwise $\left(a_{1}, a_{2}\right)=x^{\prime\left(\delta_{1}, \delta_{2}\right)}$. Hence, in the first case $\mathbf{v}=\mathbf{w}_{x}$, while in the second case $\mathbf{v}=\mathbf{w}_{x}^{\prime}$.

Suppose that $a_{1}>0$. Following Proposition [14. consider $d=d_{1} \delta_{1}+d_{2} \delta_{2}$ with $a_{1}>d_{1}>0$ and $0>d_{2}>a_{2}$.

- If $\sigma=1, w_{x_{(3)}}=a_{1} v_{1_{(3)}}+a_{2} v_{2_{(3)}}=a_{2} v_{2_{(3)}}>d_{2} v_{2_{(3)}}=d_{1} v_{1_{(3)}}+d_{2} v_{2_{(3)}}=w_{d_{(3)}}>0$. We take $i=2$, obtaining $w_{x_{(2)}}=a_{1} v_{1_{(2)}}+a_{2} v_{2_{(2)}}<d_{1} v_{1_{(2)}}+d_{2} v_{2_{(2)}}<0$.

- If $\sigma=-1, w_{x_{(3)}}=a_{1} v_{1_{(3)}}-a_{2} v_{2_{(3)}}=-a_{2} v_{2_{(3)}}<-d_{2} v_{2_{(3)}}=d_{1} v_{1_{(3)}}-d_{2} v_{2_{(3)}}=w_{d_{(3)}}<0$. Also $w_{x_{(1)}}=a_{1} v_{1_{(1)}}-a_{2} v_{2_{(1)}}>d_{1} v_{1_{(1)}}-d_{2} v_{2_{(1)}}=w_{d_{(1)}}>0$.

Now assume that $a_{1}<0$. Following Proposition 14 consider $d=d_{1}^{\prime} \delta_{1}+d_{2}^{\prime} \delta_{2}$ with $a_{1}<d_{1}^{\prime} \leq 0$ and $0<d_{2}^{\prime}<a_{2}$.

- If $\sigma=1,0>w_{d_{(3)}}^{\prime}=d_{1}^{\prime} v_{1_{(3)}}+d_{2}^{\prime} v_{2_{(3)}}=d_{2}^{\prime} v_{2_{(3)}}>a_{1} v_{1_{(3)}}+a_{2} v_{2_{(3)}}=w_{x_{(3)}}^{\prime}$. Now take $i=2$. Then $0<w_{d_{(2)}}^{\prime}=d_{1}^{\prime} v_{1_{(2)}}+d_{2}^{\prime} v_{2_{(2)}}<a_{1} v_{1_{(2)}}+a_{2} v_{2_{(2)}}=w_{x_{(2)}}^{\prime}$.

- If $\sigma=-1,0<w_{d_{(3)}}^{\prime}=d_{1}^{\prime} v_{1_{(3)}}-d_{2}^{\prime} v_{2_{(3)}}=-d_{2}^{\prime} v_{2_{(3)}}<-a_{2} v_{2_{(3)}}=a_{1} v_{1_{(3)}}-a_{2} v_{2_{(3)}}=w_{x_{(3)}}^{\prime}$. Here we choose $i=1$, obtaining $0>w_{d_{(1)}}^{\prime}=d_{1}^{\prime} v_{1_{(1)}}-d_{2}^{\prime} v_{2_{(1)}}>a_{1} v_{1_{(1)}}-a_{2} v_{2_{(1)}}=w_{x_{(1)}}^{\prime}$. 
This corollary ensures that for every $\mathbf{w}_{x}$ or $\mathbf{w}_{x}^{\prime}$ there exists a $\mathbf{w}_{d}$ or $\mathbf{w}_{d}^{\prime}$, respectively, with $d<x$ and such that the two known coordinates of $\mathbf{w}_{x}$ or $\mathbf{w}_{x}^{\prime}$, according to Table $\mathbb{1}$, are greater in absolute value than those of $\mathbf{w}_{d}$ or $\mathbf{w}_{d}^{\prime}$, respectively.

Now we are going to show how can uniqueness in Proposition 9 extend to the level of the vectors $\mathbf{w}_{x}$ and $\mathbf{w}_{x}^{\prime}$. We want to associate to each $\mathbf{v} \in M_{S}$ such that $\ell(\mathbf{v}) \in\left\{0, g, 2 g, \ldots, \max \left\{\delta_{1}, \delta_{2}\right\}\right\}$ two vectors $\mathbf{w}_{x}, \mathbf{w}_{x}^{\prime}$ such that $\ell\left(\mathbf{w}_{x}\right)=\ell\left(\mathbf{w}_{x}^{\prime}\right)=\ell(\mathbf{v})$.

Next we see that if a vector has length equal to zero, then it is a multiple of the vector appearing in Proposition 8 . Hence, when this vector is added or subtracted to another vector, the length remains the same.

Lemma 17. With the notation defined in Section 3 and Definition [10, let $\mathbf{v} \in M_{S}$ with $\ell(\mathbf{v})=0$. Then

for some $\alpha \in \mathbb{Z}$.

$$
\mathbf{v}=\alpha\left(\delta_{2} / g \mathbf{v}_{1}-\sigma \delta_{1} / g \mathbf{v}_{2}\right)
$$

Proof. Since $\left\{\mathbf{v}_{1}, \sigma \mathbf{v}_{2}\right\}$ is a basis for $M_{S}$, we have that there exists $\lambda_{1}, \lambda_{2} \in \mathbb{Z}$ such that $\mathbf{v}=$ $\lambda_{1} \mathbf{v}_{1}+\lambda_{2} \sigma \mathbf{v}_{2}$. Now $0=\ell(\mathbf{v})=\lambda_{1} \delta_{1}+\lambda_{2} \delta_{2}$. Since $\operatorname{gcd}\left(\delta_{1}, \delta_{2}\right)=g$, we deduce that $\delta_{1} / g$ divides $\lambda_{2}$, and $\delta_{2} / g$ divides $\lambda_{1}$. So there exists $k_{1}$ and $k_{2}$ integers such that $\lambda_{1}=k_{1} \delta_{2} / g$ and $\lambda_{2}=k_{2} \delta_{1} / g$. We then have $0=k_{1}+k_{2}$ and consequently $k_{1}=-k_{2}$. Take $\alpha=k_{1} / g$. Hence $\mathbf{v}=\alpha \delta_{2} \mathbf{v}_{1}-\alpha \delta_{1} \sigma \mathbf{v}_{2}$.

We now characterize the vectors in $M_{S}$ with length in $\left\{1, \ldots, \max \left\{\delta_{1}, \delta_{2}\right\}\right\}$.

Proposition 18. Under the hypotheses and notations of Section 3 and Definition 10, let $\mathbf{v} \in M_{S}$ with $0<\ell(\mathbf{v}) \leq \max \left\{\delta_{1}, \delta_{2}\right\}$. Then

$$
\mathbf{v}=a_{1} \mathbf{v}_{1}+\sigma a_{2} \mathbf{v}_{2}+\alpha\left(\delta_{2} / g \mathbf{v}_{1}-\sigma \delta_{1} / g \mathbf{v}_{2}\right),
$$

for some $a_{1}, a_{2} \in \mathbb{Z}^{2} \backslash\{(0,0)\}$ such that $a_{1} a_{2} \leq 0,-\delta_{2} / g<a_{1} \leq \delta_{2} / g,-\delta_{1} / g<a_{2} \leq \delta_{1} / g$, where $g=\operatorname{gcd}\left(\delta_{1}, \delta_{2}\right)$ and $\alpha \in \mathbb{Z}$.

Furthermore, $a_{1}, a_{2}$ and $\alpha$ are unique if we impose that the third coordinates of $\mathbf{u}=a_{1} \mathbf{v}_{1}+\sigma a_{2} \mathbf{v}_{2}$ and $\mathbf{v}$ have the same sign.

Proof. We use once more that $\left\{\mathbf{v}_{1}, \sigma \mathbf{v}_{2}\right\}$ is a generating system for $M_{S}$; then there exist unique $\lambda_{1}, \lambda_{2} \in \mathbb{Z}$ such that $\mathbf{v}=\lambda_{1} \mathbf{v}_{1}+\lambda_{2} \sigma \mathbf{v}_{2}$.

Let us prove that $\lambda_{1} \lambda_{2} \leq 0$. Clearly, if $\lambda_{1} \lambda_{2}=0$, we are done.

Assume that $\lambda_{1}>0$ (the other case is analogous).

- If $\delta_{1}>\delta_{2}$, we have $0<\ell(\mathbf{v})=\lambda_{1} \delta_{1}+\lambda_{2} \delta_{2} \leq \max \left\{\delta_{1}, \delta_{2}\right\}=\delta_{1}$. Then $\lambda_{2} \delta_{2} \leq\left(1-\lambda_{1}\right) \delta_{1} \leq 0$, which implies $\lambda_{2} \leq 0$.

- If $\delta_{2}>\delta_{1}$, we have $0<\ell(\mathbf{v})=\lambda_{1} \delta_{1}+\lambda_{2} \delta_{2} \leq \max \left\{\delta_{1}, \delta_{2}\right\}=\delta_{2}$. Then $0<\lambda_{1} \delta_{1} \leq\left(1-\lambda_{2}\right) \delta_{2}$ and $0<1-\lambda_{2}$, yielding $\lambda_{2} \leq 0$.

Next, we prove the following assertions.

$$
\begin{array}{lll}
\lambda_{1}>\delta_{2} / g & \text { implies } & \lambda_{2} \leq-\delta_{1} / g \\
\lambda_{1} \leq-\delta_{2} / g & \text { implies } & \lambda_{2}>\delta_{1} / g \\
-\delta_{2} / g<\lambda_{1} \leq 0 & \text { implies } & 0<\lambda_{2} \leq \delta_{1} / g \\
0<\lambda_{1} \leq \delta_{2} / g & \text { implies } & -\delta_{1} / g<\lambda_{2} \leq 0
\end{array}
$$

(1) Assume that $\lambda_{1}>\delta_{2} / g$.

- If $\delta_{1}>\delta_{2}$, we have that $0<\ell(\mathbf{v})=\lambda_{1} \delta_{1}+\lambda_{2} \delta_{2} \leq \delta_{1}$. Hence $\lambda_{2} \delta_{2} \leq\left(1-\lambda_{1}\right) \delta_{1}$. From $\lambda_{1}>\delta_{2} / g$, we deduce $\left(1-\lambda_{1}\right) \leq-\delta_{2} / g$. Therefore, $\lambda_{2} \delta_{2} \leq\left(1-\lambda_{1}\right) \delta_{1} \leq-\delta_{1} \delta_{2} / g$, and thus $\lambda_{2} \leq-\delta_{1} / g$.

- If $\delta_{2}>\delta_{1}$, we have $0<\ell(\mathbf{v})=\lambda_{1} \delta_{1}+\lambda_{2} \delta_{2} \leq \delta_{2}$, and then $\lambda_{1} \delta_{1} \leq\left(1-\lambda_{2}\right) \delta_{2}$. As $\delta_{2} / g<\lambda_{1}$, we get $\delta_{2} \delta_{1} / g<\lambda_{1} \delta_{1}$. This implies $\delta_{1} / g<\left(1-\lambda_{2}\right)$ or equivalently $\lambda_{2} \leq-\delta_{1} / g$. 
(2) Assume now that $\lambda_{1} \leq-\delta_{2} / g$. Then $0<\ell(\mathbf{v})=\lambda_{1} \delta_{1}+\lambda_{2} \delta_{2} \leq-\delta_{2} \delta_{1} / g+\lambda_{2} \delta_{2}=\left(\lambda_{2}-\delta_{1} / g\right) \delta_{2}$. This forces $\lambda_{2}>\delta_{1} / g$.

(3) Suppose that $-\delta_{2} / g<\lambda_{1} \leq 0$.

- If $\delta_{1}>\delta_{2}$, then $0<\ell(\mathbf{v})=\lambda_{1} \delta_{1}+\lambda_{2} \delta_{2} \leq \delta_{1}$. As $\left(1-\lambda_{1}\right) \leq \delta_{2} / g$, we deduce that $\lambda_{2} \delta_{2} \leq\left(1-\lambda_{1}\right) \delta_{1} \leq \delta_{1} \delta_{2} / g$, and consequently $\lambda_{2} \leq \delta_{1} / g$.

- If $\delta_{2}>\delta_{1}$, we have $\ell(\mathbf{v})=\lambda_{1} \delta_{1}+\lambda_{2} \delta_{2} \leq \delta_{2}$. Since $-\delta_{2} / g<\lambda_{1}$, we obtain $-\delta_{2} \delta_{1} / g+\lambda_{2} \delta_{2}<$ $\lambda_{1} \delta_{1}+\lambda_{2} \delta_{2} \leq \delta_{2}$. Hence $\lambda_{2}-\delta_{1} / g<1$, or equivalently, $\lambda_{2} \leq \delta_{1} / g$.

(4) Finally, assume that $0<\lambda_{1} \leq \delta_{2} / g$. Then $0<\ell(\mathbf{v})=\lambda_{1} \delta_{1}+\lambda_{2} \delta_{2} \leq \delta_{2} \delta_{1} / g+\lambda_{2} \delta_{2}=$ $\left(\lambda_{2}+\delta_{1} / g\right) \delta_{2}$. Hence $-\delta_{1} / g<\lambda_{2}$.

In order to conclude the proof it suffices to observe the following.

- If $\lambda_{1}$ fulfills the conditions (3) or (4), we are done.

- If $\lambda_{1}>\delta_{2} / g$, take $\alpha \in \mathbb{Z}$ such that $0<\lambda_{1}-\alpha \delta_{2} / g \leq \delta_{2} / g$. Set $a_{1}=\lambda_{1}-\alpha \delta_{2} / g \leq \delta_{2} / g$ and $a_{2}=\lambda_{2}+\alpha \delta_{1} / g$. Define $\mathbf{u}=a_{1} \mathbf{v}_{1}+\sigma a_{2} \mathbf{v}_{2}$. Then $\ell(\mathbf{v})=\ell(\mathbf{u})$, and $\mathbf{v}=\mathbf{u}+\alpha\left(\delta_{2} / g \mathbf{v}_{1}-\right.$ $\left.\sigma \delta_{1} / g \mathbf{v}_{2}\right)$. Now $\mathbf{u}$ fulfills (4), and we are done. Notice that if we take $\alpha+1$ instead of $\alpha$, we fall in case (3), with $\mathbf{u}^{\prime}=\mathbf{u}-\left(\delta_{2} / g \mathbf{v}_{1}-\sigma \delta_{1} / g \mathbf{v}_{2}\right)$ : thus it can be deduced that there are two possible choices of $\alpha$ fulfilling the conditions of the statement.

- If $\lambda_{1}<-\delta_{2} / g$, we proceed analogously.

Since there are two possible choices of $\alpha$, but $\lambda_{1}$ and $\lambda_{2}$ are unique, there exist two possible choices for $\left(a_{1}, a_{2}\right)$. In order to conclude the proof, it suffices to show that the signs of the third coordinates of $\mathbf{u}$ and $\mathbf{u}^{\prime}$ are different (and thus one of them must be equal to the sign of the third coordinate of $\mathbf{v})$. Assume that $\alpha$ is such that $0<a_{1} \leq \delta_{2} / g$, and thus by (4) applied to $\mathbf{u},-\delta_{1} / g<\sigma a_{2} \leq 0$.

If we write $\mathbf{u}^{\prime}=a_{1}^{\prime} \mathbf{v}_{1}+\sigma a_{2}^{\prime} \mathbf{v}_{2}$ then, as $a_{1}^{\prime}=a_{1}-\delta_{2} / g>0$, by (3) we have $\sigma a_{2}^{\prime}>0$. Since $a_{2}$ and $a_{2}^{\prime}$ have different signs, we are done.

This proposition will be used later to work with $\mathbf{u}$ instead of $\mathbf{v}$.

The goal of this work is to prove the following theorem. For clarity's sake, we first give an example; then, we provide the intermediate results needed for its proof, which will be a direct consequence of Lemmas 22 and 24.

Theorem 19. Let $S$ be a symmetric numerical semigroup with embedding dimension three. With the notation introduced in Section 13 and Definition 10, we have

$$
\Delta(S)=\operatorname{Euc}\left(\delta_{1}, \delta_{2}\right) \backslash\{0\} .
$$

Example 20. Let $S=\left\langle s_{2} s_{3}, s_{1} s_{3}, s_{1} s_{2}\right\rangle=\langle 2015,7124,84940\rangle$, with $s_{1}=548, s_{2}=155$, and $s_{3}=13$. Then $\mathbf{v}_{1}=(548,-155,0)$ and $\mathbf{v}_{2}=(0,155,-13)$. Hence $\delta_{1}=393$ and $\delta_{2}=142$. Extended Euclid's Algorithm for $\delta_{1}=393$ and $\delta_{2}=142$ yields the Table 20, whose cells contain the vectors $\mathbf{w}_{d}$ or $\mathbf{w}_{d}^{\prime}$, as defined in (11), for $d \in \operatorname{Euc}\left(\delta_{1}, \delta_{2}\right)$.

According to Theorem 19, we would obtain

$$
\Delta(S)=\{1,2,3,4,7,10,13,23,33,43,76,109,142,251,393\} .
$$

Lemma 21. Let $\mathbf{z}, \mathbf{z}^{\prime} \in \mathrm{Z}(s)$, with $0 \neq s \in S$. With the notation introduced in Section 3, if $0<\ell(\mathbf{z})-\ell\left(\mathbf{z}^{\prime}\right)<\max \left\{\delta_{1}, \delta_{2}\right\}$, then

(1) $\mathbf{z}-\mathbf{z}^{\prime}$ is either of the form $(?,+,-)$ or $(?,-,+)$ when $\ell\left(\mathbf{v}_{2}\right)>0(\sigma=1)$, and

(2) $\mathbf{z}-\mathbf{z}^{\prime}$ is of the form $(+, ?,-)$ or $(-, ?,+)$ when $\ell\left(\mathbf{v}_{2}\right)<0(\sigma=-1)$.

Proof. Recall that $\mathbf{z}, \mathbf{z}^{\prime} \in \mathbb{N}^{3}$. Since $\mathbf{z}-\mathbf{z}^{\prime} \in M_{S}$ and $0<\ell\left(\mathbf{z}-\mathbf{z}^{\prime}\right) \leq \max \left\{\delta_{1}, \delta_{2}\right\}$ by Proposition 18 we know that $\mathbf{z}-\mathbf{z}^{\prime}=a_{1} \mathbf{v}_{1}+\sigma a_{2} \mathbf{v}_{2}+\alpha\left(\delta_{2} / g \mathbf{v}_{1}-\sigma \delta_{1} / g \mathbf{v}_{2}\right)$. If $\alpha>0$, we have that $\mathbf{z}-\mathbf{z}^{\prime}=$ $\left(\alpha \delta_{2} / g+a_{1}\right) \mathbf{v}_{1}+\sigma\left(a_{2}-\alpha \delta_{1} / g\right) \mathbf{v}_{2}$, and clearly $\left(\alpha \delta_{2} / g+a_{1}\right)>0$ and $\left(a_{2}-\alpha \delta_{1}\right) / g<0$. According to Table 1, $\mathbf{z}-\mathbf{z}^{\prime}=(?,-,+)$ if $\sigma=1$, or $\mathbf{z}-\mathbf{z}^{\prime}=(+, ?,-)$ if $\sigma=-1$. A similar argument shows that if $\alpha<0$, then $\mathbf{z}-\mathbf{z}^{\prime}=(?,+,-)$ for $\sigma=1$, and $\mathbf{z}-\mathbf{z}^{\prime}=(-, ?,+)$ for $\sigma=-1$.

For the case $\alpha=0$, as $a_{1} a_{2}<0$ there are two possibilities. 


\begin{tabular}{|c|c|c|c|}
\hline $\begin{array}{l}\mathbf{w}_{d}=(?,-,+) \\
\mathrm{D}\left(\delta_{1}, \delta_{2}\right)\end{array}$ & $\begin{aligned} \mathbf{w}_{393}= & \mathbf{v}_{1} \\
= & (548,-155,0) \\
& \delta_{1}=393\end{aligned}$ & $\begin{aligned} \mathbf{w}_{251} & =\mathbf{v}_{1}-\mathbf{v}_{2} \\
& =(548,-310,13) \\
& \delta_{1}-\delta_{2}=251\end{aligned}$ & $\begin{array}{c}\mathbf{w}_{109}=\mathbf{v}_{1}-2 \mathbf{v}_{2} \\
=(548,-465,26) \\
\delta_{1}-2 \delta_{2}=109\end{array}$ \\
\hline $\begin{array}{l}\mathbf{w}_{d}^{\prime}=(?,+,-) \\
\mathrm{D}\left(\delta_{2}, \delta_{3}\right)\end{array}$ & $\begin{aligned} \mathbf{w}_{142}^{\prime} & =\mathbf{v}_{2} \\
& =(0,155,-13) \\
& \delta_{2}=142\end{aligned}$ & $\begin{aligned} \mathbf{w}_{33}^{\prime}=-\mathbf{v}_{1}+3 \mathbf{v}_{2} \\
=(-548,620,-39) \\
\delta_{2}-\delta_{3}=33\end{aligned}$ & \\
\hline $\begin{array}{l}\mathbf{w}_{d}=(?,-,+) \\
\mathrm{D}\left(\delta_{3}, \delta_{4}\right)\end{array}$ & $\begin{aligned} \mathbf{w}_{76}= & \mathbf{2} v_{1}-5 \mathbf{v}_{2} \\
= & (1096,-1085,65) \\
& \delta_{3}-\delta_{4}=76\end{aligned}$ & $\begin{aligned} \mathbf{w}_{43}= & \mathbf{3} \mathbf{v}_{1}-8 \mathbf{v}_{2} \\
= & (1644,-1705,104) \\
& \delta_{3}-2 \delta_{4}=43\end{aligned}$ & $\begin{aligned} \mathbf{w}_{10}= & 4 \mathbf{v}_{1}-11 \mathbf{v}_{2} \\
= & (2192,-2325,143) \\
& \delta_{3}-3 \delta_{4}=10\end{aligned}$ \\
\hline $\begin{array}{l}\mathbf{w}_{d}^{\prime}=(?,+,-) \\
\mathrm{D}\left(\delta_{4}, \delta_{5}\right)\end{array}$ & $\begin{aligned} \mathbf{w}_{23}^{\prime}= & -5 \mathbf{v}_{1}+14 \mathbf{v}_{2} \\
= & (-2740,2945,-182) \\
& \delta_{4}-\delta_{5}=23\end{aligned}$ & $\begin{aligned} \mathbf{w}_{13}^{\prime}= & -9 \mathbf{v}_{1}+25 \mathbf{v}_{2} \\
= & (-4932,5270,-325) \\
& \delta_{4}-2 \delta_{5}=13\end{aligned}$ & $\begin{array}{c}\mathbf{w}_{3}^{\prime}=-13 \mathbf{v}_{1}+36 \mathbf{v}_{2} \\
(-7124,7595,-468) \\
\delta_{4}-3 \delta_{5}=3\end{array}$ \\
\hline $\begin{array}{l}\mathbf{w}_{d}=(?,-,+) \\
\mathrm{D}\left(\delta_{5}, \delta_{6}\right)\end{array}$ & $\begin{aligned} \mathbf{w}_{7}= & 17 \mathbf{v}_{1}-47 \mathbf{v}_{2} \\
= & (9316,-9920,611) \\
& \delta_{5}-\delta_{6}=7\end{aligned}$ & $\begin{aligned} \mathbf{w}_{4}= & 30 \mathbf{v}_{1}-83 \mathbf{v}_{2} \\
= & (16440,-17515,1079) \\
& \delta_{5}-2 \delta_{6}=4\end{aligned}$ & $\begin{aligned} \mathbf{w}_{1}= & 43 \mathbf{v}_{1}-119 \mathbf{v}_{2} \\
= & (23564,-25110,1547) \\
& \delta_{5}-3 \delta_{6}=1\end{aligned}$ \\
\hline $\begin{array}{l}\mathbf{w}_{d}^{\prime}=(?,+,-) \\
\mathrm{D}\left(\delta_{6}, \delta_{7}\right)\end{array}$ & $\begin{aligned} & =-56 \mathbf{v}_{1}+155 \mathbf{v}_{2} \\
= & (-30688,32705,-2015) \\
& \delta_{6}-\delta_{7}=2\end{aligned}$ & $\begin{aligned} \mathbf{w}_{1}=-99 \mathbf{v}_{1}+274 \mathbf{v}_{2} \\
=(-54252,57815,-3562) \\
\quad \delta_{6}-2 \delta_{7}=1\end{aligned}$ & $\begin{aligned} \mathbf{w}_{0}=-142 \mathbf{v}_{1}+393 \mathbf{v}_{2} \\
=(-77816,82925,-5109) \\
\quad \delta_{6}-3 \delta_{7}=0\end{aligned}$ \\
\hline
\end{tabular}

TABLE 2. Table for $\langle 2015,7124,84940\rangle$

- If $a_{1}<0$ and $a_{2}>0$, then $\mathbf{z}-\mathbf{z}^{\prime}=($ ?,,+-$)$ for $\sigma=1$, and $\mathbf{z}-\mathbf{z}^{\prime}=(-, ?,+)$ for $\sigma=-1$.

- If $a_{1}>0$ and $a_{2}<0$, then $\mathbf{z}-\mathbf{z}^{\prime}=(?,-,+)$ for $\sigma=1$, and $\mathbf{z}-\mathbf{z}^{\prime}=(+, ?,-)$ for $\sigma=-1$.

Lemma 22. Let $S=\left\langle n_{1}, n_{2}, n_{3}\right\rangle$ be a symmetric numerical semigroup with embedding dimension three. With the notation introduced in Section 3 and Definition 10 , let $\mathbf{z}, \mathbf{z}^{\prime} \in \mathbf{Z}(s)$, for some $s \in S \backslash\{0\}$ such that $x=\ell(\mathbf{z})-\ell\left(\mathbf{z}^{\prime}\right) \notin \operatorname{Euc}\left(\delta_{1}, \delta_{2}\right)$. Then there exists $\mathbf{z}^{\prime \prime} \in \mathbf{Z}(s)$ such that $\ell\left(\mathbf{z}^{\prime}\right)<\ell\left(\mathbf{z}^{\prime \prime}\right)<\ell(\mathbf{z})$.

Proof. If $z_{(i)} \cdot z_{(i)}^{\prime} \neq 0$, we consider $s^{\prime}=s-\left|z_{(i)}-z_{(i)}^{\prime}\right| n_{i}$, which is an element in $S$ with two factorizations $\mathbf{z}$ and $\mathbf{z}^{\prime}$ with $\ell(\mathbf{z})-\ell\left(\mathbf{z}^{\prime}\right)=x$ and so that one of them has the $i^{\text {th }}$ coordinate equal to zero. With this argument, we can assume $\mathbf{z} \cdot \mathbf{z}^{\prime}=0$. Later, by adding $\sum_{i=1}^{3}\left|z_{(i)}-z_{(i)}^{\prime}\right| \mathbf{e}_{i}\left(\mathbf{e}_{i}\right.$ is the $i^{\text {th }}$ row of the $3 \times 3$ identity matrix) to the factorizations obtained, we will recover the original factorizations.

Now, from Proposition 18, we can write $\mathbf{z}-\mathbf{z}^{\prime}=a_{1} \mathbf{v}_{1}+\sigma a_{2} \mathbf{v}_{2}+\alpha\left(\delta_{2} / g \mathbf{v}_{1}-\sigma \delta_{1} / g \mathbf{v}_{2}\right)$ and $\mathbf{u}=a_{1} \mathbf{v}_{1}+\sigma a_{2} \mathbf{v}_{2}$ in such a way that $\mathbf{z}-\mathbf{z}^{\prime}=\left(z_{(1)}-z_{(1)}^{\prime}, z_{(2)}-z_{(2)}^{\prime}, z_{(3)}-z_{(3)}^{\prime}\right), \mathbf{u}=\left(u_{(1)}, u_{(2)}, u_{(3)}\right)$ and $\alpha\left(\delta_{2} / g \mathbf{v}_{1}-\sigma \delta_{1} / g \mathbf{v}_{2}\right)=\left(\alpha_{(1)}, \alpha_{(2)}, \alpha_{(3)}\right)$. So, we have for $j \in\{1,2,3\}, z_{(j)}-z_{(j)}^{\prime}=u_{(j)}+\alpha_{(j)}$. As the sign of the third coordinates of $\mathbf{z}-\mathbf{z}^{\prime}$ and $\mathbf{u}$ is the same and the third coordinate of $\mathbf{u}$ is smaller in absolute value, we necessarily deduce that $\alpha_{(3)}$ has the same sign too. Looking on Table 1 and Proposition 8 we have $\operatorname{sgn}\left(z_{(i)}-z_{(i)}^{\prime}\right)=\operatorname{sgn}\left(u_{(i)}\right)=\operatorname{sgn}\left(\alpha_{(i)}\right)$ and $\operatorname{sgn}\left(z_{(j)}-z_{(j)}^{\prime}\right)=\operatorname{sgn}\left(u_{(j)}\right)=$ $\operatorname{sgn}\left(\alpha_{(j)}\right)$, for some $i \in\{1,2\}$ and $j=3$,

In light of Proposition 9, if $-\delta_{2} / g<a_{1} \leq 0<a_{2} \leq \delta_{1} / g$, then $-\delta_{1} / g<a_{2}-\delta_{1} / g \leq 0<$ $a_{1}+\delta_{2} / g \leq \delta_{2} / g$. According to the different cases in Corollary [16, we obtain the following.

(1) If $a_{1}>0$, then $\mathbf{v}=\left(v_{(1)}, v_{(2)}, v_{(3)}\right)=\mathbf{w}_{d}$ for $d<\ell\left(\mathbf{z}-\mathbf{z}^{\prime}\right)=\ell(\mathbf{u})$.

(2) If $a_{1}<0$, then $\mathbf{v}=\left(v_{(1)}, v_{(2)}, v_{(3)}\right)=\mathbf{w}_{d}^{\prime}$ for $d<\ell\left(\mathbf{z}-\mathbf{z}^{\prime}\right)=\ell(\mathbf{u})$.

Now we have that $0<\ell(\mathbf{v})=d<\ell(\mathbf{z})-\ell\left(\mathbf{z}^{\prime}\right)$. By adding $\ell\left(\mathbf{z}^{\prime}\right)$ we obtain $\ell\left(\mathbf{z}^{\prime}\right)<\ell(\mathbf{v})+\ell\left(\mathbf{z}^{\prime}\right)<$ $\ell(\mathbf{z})$, and it can be easily deduced that $0<\ell\left(\mathbf{z}^{\prime}\right)<\ell(\mathbf{z})-\ell(\mathbf{v})<\ell(\mathbf{z})$.

If all the coordinates of $\mathbf{v}+\mathbf{z}^{\prime}$ are positive, or all the coordinates of $\mathbf{z}-\mathbf{v}$ are positive, then we would obtain a factorization of $s$ with length comprised between $\ell\left(\mathbf{z}^{\prime}\right)$ and $\ell(\mathbf{z})$ : thus we only have to prove that either $\mathbf{z}-\mathbf{v}$ or $\mathbf{v}+\mathbf{z}^{\prime}$ has positive coordinates. 
By Proposition 14 and the behavior of the coordinates described in Corollary 16, we can deduce that two of the three coordinates (the second and third if $\ell\left(\mathbf{v}_{2}\right)>0$, and the first and third if $\ell\left(\mathbf{v}_{2}\right)<0$ ) of $\mathbf{v}$ are smaller in absolute value than the corresponding coordinates of $\mathbf{u}$. Thus we have two possible cases, according to the coordinate whose sign we do not control (the first if $\ell\left(\mathbf{v}_{2}\right)>0$, and the second if $\left.\ell\left(\mathbf{v}_{2}\right)<0\right)$.

Assume first that this coordinate is negative. In this setting, the corresponding coordinate of $\mathbf{z}-\mathbf{v}$ is positive, while for the other two we have:

- if $v_{(j)}<0$, for some $j$, as $z_{(j)} \geq 0$ we have that the $j^{\text {th }}$ coordinate of $\mathbf{z}-\mathbf{v}$ will be positive, and

- if $v_{(j)}>0$, we know that $u_{(j)}>v_{(j)}>0$ and, $\operatorname{as~} \operatorname{sgn}\left(z_{(j)}-z_{(j)}^{\prime}\right)=\operatorname{sgn}\left(u_{(j)}\right)=\operatorname{sgn}\left(\alpha_{(j)}\right)$ and $z_{(j)}-z_{(j)}^{\prime}-\alpha_{(j)}=u_{(j)}>v_{(j)}$ we have that $z_{(j)}-v_{(j)}>0$, and thus the $j^{\text {th }}$ coordinate $\mathbf{z}-\mathbf{v}$ would be again positive.

If the above unknown coordinate is positive, then we take $\mathbf{v}+\mathbf{z}^{\prime}$ instead of $\mathbf{z}-\mathbf{v}$, and make the same coordinate positive. For the other two coordinates, if $v_{(j)}>0$, we do nothing, and if $v_{(j)}<0$, as $0<-v_{(j)}<-u_{(j)}=-z_{(j)}+z_{(j)}^{\prime}+\alpha_{(j)} \leq z_{(j)}^{\prime}$, remember that in this case $\alpha_{(j)}$ is negative. As $z_{(j)}^{\prime}$ is the $j$-th coordinate of $\mathbf{z}^{\prime}$ we would again have that $z_{(j)}^{\prime}+v_{(j)}$ is a positive coordinate, and by adding $\ell\left(\mathbf{z}^{\prime}\right)$ we obtain that $\ell\left(\mathbf{z}^{\prime}\right)<\ell\left(\mathbf{v}+\mathbf{z}^{\prime}\right)<\ell(\mathbf{z})$.

Example 23. In the setting of Examples11and 15, take $11630 n_{2} \in S$ and consider $\mathbf{z}=(10960,5,715)$ and $\mathbf{z}^{\prime}=(0,11603,0)$. We have $\ell(\mathbf{z})=11680$ and $\ell\left(\mathbf{z}^{\prime}\right)=11630$, so $\ell\left(\mathbf{z}-\mathbf{z}^{\prime}\right)=50<\max \left\{\delta_{1}, \delta_{2}\right\}$. As $z_{(2)} \cdot z_{(2)}^{\prime} \neq 0$, we can consider $s^{\prime}=11630 n_{2}-5 n_{2}$ as the $s^{\prime}$ in the proof of Lemma 22. So, we are going to work with $s^{\prime}=11625 n_{2}, \mathbf{z}=(10960,0,715)$ and $\mathbf{z}^{\prime}=(0,11625,0)$, obtaining $\mathbf{z}-\mathbf{z}^{\prime}=(10960,-11625,715)=20 \mathbf{v}_{1}-5 \mathbf{v}_{2}$. In this case $\alpha=0$, whence $\mathbf{u}=20 \mathbf{v}_{1}-5 \mathbf{v}_{2}$. As we showed in Example 15, we can consider $\mathbf{w}_{d}=\mathbf{w}_{43}=3 \mathbf{v}_{1}-8 \mathbf{v}_{2}=(1644,-1705,104)$. Now, if we consider $\mathbf{w}_{d}+\mathbf{z}^{\prime}=(1644,9920,104)$, we obtain a new factorization of $11625 n_{2}$ with $11630=$ $\ell\left(\mathbf{z}^{\prime}\right)<\ell\left(\mathbf{w}_{d}+\mathbf{z}^{\prime}\right)=11668<\ell(\mathbf{z})=11680$, while if we consider $\mathbf{z}-\mathbf{w}_{d}=(9316,1705,611)$, a new factorization of $11625 n_{2}$ can be obtained, with $\ell\left(\mathbf{z}-\mathbf{w}_{d}\right)=11632$ which, again, is between $\ell\left(\mathbf{z}^{\prime}\right)$ and $\ell(\mathbf{z})$. In this case, both factorizations can be chosen. Finally adding 5 to the second coordinate, we obtain factorizations of the original element, $11630 n_{2}$.

Now if we choose $71300 n_{2} \in S$, we have two factorizations $\mathbf{z}=(66856,0,4394)$ and $\mathbf{z}^{\prime}=$ $(0,71300,0)$ whose lengths are $\ell(\mathbf{z})=71250$ and $\ell\left(\mathbf{z}^{\prime}\right)=71300$, obtaining again that $\ell\left(\mathbf{z}^{\prime}\right)-\ell(\mathbf{z})=$ 50. In this case $\mathbf{u}=-122 \mathbf{v}_{1}+338 \mathbf{v}_{2}$ because $\mathbf{z}^{\prime}-\mathbf{z}$ is of the type $(?,+,-)$ so $a_{1}<0$ and $a_{2}>0$. According to Example 15, we can pick $\mathbf{w}_{33}^{\prime}=-\mathbf{v}_{1}+3 \mathbf{v}_{2}=(-548,620,-39)$ to obtain $\mathbf{w}_{d}^{\prime}+\mathbf{z}=(66308,620,4355)$ and $\mathbf{z}^{\prime}-\mathbf{w}_{d}^{\prime}=(548,70680,39)$; hence we obtained two factorizations of $71300 n_{2}$ whose lengths $\ell\left(\mathbf{w}_{d}^{\prime}+\mathbf{z}\right)=71283$ and $\ell\left(\mathbf{z}^{\prime}-\mathbf{w}_{d}^{\prime}\right)=71267$ are both between $\ell(\mathbf{z})$ and $\ell\left(\mathbf{z}^{\prime}\right)$.

Lemma 24. Let $S=\left\langle n_{1}, n_{2}, n_{3}\right\rangle$ be a symmetric numerical semigroup with embedding dimension three. With the notation introduced in Section 3 and Definition 10, consider $d \in \operatorname{Euc}\left(\delta_{1}, \delta_{2}\right)$. Then there exists $s \in S$ and $\mathbf{z}, \mathbf{z}^{\prime} \in \mathbf{Z}(s)$, with $d=\ell(\mathbf{z})-\ell\left(\mathbf{z}^{\prime}\right)$, and such that for any other $\mathbf{z}^{\prime \prime} \in \mathbf{Z}(s)$ either $\ell\left(\mathbf{z}^{\prime \prime}\right) \leq \ell\left(\mathbf{z}^{\prime}\right)$ or $\ell\left(\mathbf{z}^{\prime \prime}\right) \geq \ell(\mathbf{z})$.

Proof. Let $d \in \operatorname{Euc}\left(\delta_{1}, \delta_{2}\right)$, and set $\mathbf{v}=x_{1} \mathbf{v}_{1}+\sigma x_{2} \mathbf{v}_{2}$, with $x_{1} x_{2}<0$, and $-\delta_{1} / g<x_{2} \leq \delta_{1} / g$ and $-\delta_{2} / g<x_{1} \leq \delta_{2} / g$, such that $\ell(\mathbf{v})=d$. Then

$$
\mathbf{v}=\left(x_{1} v_{1_{(1)}}+\sigma x_{2} v_{2_{(1)}}, x_{1} v_{1_{(2)}}+\sigma x_{2} v_{2_{(2)}},-\sigma x_{2} v_{2_{(3)}}\right),
$$

with $\ell(\mathbf{v})=d>0$. Clearly, $\mathbf{v}$ has two coordinates with the same sign, and the other with opposite sign. Let us denote the latter by $i$.

Set $s=\left|v_{x_{(i)}}\right| n_{i}$, and $\mathbf{z}=\left(v_{x_{(1)}}^{+}, v_{x_{(2)}}^{+}, v_{x_{(3)}}^{+}\right)$and $\mathbf{z}^{\prime}=\left(v_{x_{(1)}}^{-}, v_{x_{(2)}}^{-}, v_{x_{(3)}}^{-}\right)$, where $a^{+}=(|a|+a) / 2 \geq 0$ and $a^{-}=(|a|-a) / 2 \geq 0$. Then $\mathbf{z}$ and $\mathbf{z}^{\prime}$ are factorizations of $s$. The rest of the factorizations of $s$ are of the form $\mathbf{z}^{\prime \prime}=\mathbf{z}^{\prime}+a_{1} \mathbf{v}_{1}+a_{2} \sigma \mathbf{v}_{2}$, with $a_{1} a_{2}<0$. We are concerned with those such 
that $0<a_{1} \delta_{1}+a_{2} \delta_{2}<d$, in order to find one with length between $\ell(\mathbf{z})$ and $\ell\left(\mathbf{z}^{\prime}\right)$. Denote $\mathbf{u}=$ $a_{1} \mathbf{v}_{1}+a_{2} \sigma \mathbf{v}_{2}=\left(u_{(1)}, u_{(2)}, u_{(3)}\right)$.

Next, we prove that $\left|a_{1}\right| \geq\left|x_{1}\right|$ and $\left|a_{2}\right|>\left|x_{2}\right|$.

Assume that $\ell(\mathbf{u}) \in \operatorname{Euc}\left(\delta_{1}, \delta_{2}\right)$. Then, as $\ell(\mathbf{u})<\ell(\mathbf{v})$, by construction (see Remark 12) $\left|a_{1}\right| \geq\left|x_{1}\right|$ and $\left|a_{2}\right|>\left|x_{2}\right|$. On the other hand, if $\ell(\mathbf{u}) \notin \operatorname{Euc}\left(\delta_{1}, \delta_{2}\right)$, then we consider $e=\operatorname{bsm}(\ell(\mathbf{u})) \in$ $\operatorname{Euc}\left(\delta_{1}, \delta_{2}\right)$ associated to $\mathbf{u}$ (Proposition 14), and then we have $\left|a_{1}\right|>\left|e_{1}\right|>\left|x_{1}\right|$ and $\left|a_{2}\right|>\left|e_{2}\right|>$ $\left|x_{2}\right|$, and the assertion is proved.

Thus, Table 3 collects the different possible settings.

\begin{tabular}{c|cc} 
& $\sigma=1$ & $\sigma=-1$ \\
& $\mathbf{v}_{1}=(+,-, 0), \mathbf{v}_{2}=(+,+,-)$ & $\mathbf{v}_{1}=(+,-, 0), \sigma \mathbf{v}_{2}=(-,-,+)$ \\
\hline$x_{1}>0, x_{2}<0$ & $\mathbf{v}=(?,-,+)$ & $\mathbf{v}=(+, ?,-)$ \\
$x_{1}^{\prime}<0, x_{2}^{\prime}>0$ & $\mathbf{v}=(?,+,-)$ & $\mathbf{v}=(-, ?,+)$ \\
$a_{1}>0, a_{2}<0$ & $\mathbf{u}=(?,-,+)$ & $\mathbf{u}=(+, ?,-)$ \\
$a_{1}^{\prime}<0, a_{2}^{\prime}>0$ & $\mathbf{u}=(?,+,-)$ & $\mathbf{u}=(-, ?,+)$
\end{tabular}

TABLE 3. Signs for $\mathbf{v}$ and $\mathbf{u}$

According to Table 3, we have the following cases.

(1) If $\sigma=1$, we obtain that $\left|u_{(2)}\right|>\left|v_{(2)}\right|$ and $\left|u_{(3)}\right|>\left|v_{(3)}\right|$

(a) If $\mathbf{v}=(?,-,+)$, then $z_{(2)}=0$, and $z_{(2)}^{\prime} \leq 0$. If in addition $u_{(2)}<0$, then $z_{(2)}^{\prime \prime}<0$, which is a contradiction; and if $u_{(2)}>0$ we derive $u_{(3)}<0$ and as $\left|u_{3}\right|=\left|a_{2}\right|\left|v_{2_{(3)}}\right|>$ $\left|x_{2}\right|\left|v_{2_{(3)}}\right|=\left|v_{(3)}\right|$, we obtain $z_{(3)}^{\prime \prime}<0$, which is another contradiction.

(b) If $\mathbf{v}=(?,+,-)$, then $z_{(3)}=0$. If $u_{(3)}<0$, then $z_{(3)}^{\prime \prime}<0$, a contradiction. If $u_{(3)}>$ 0 , we have that $u_{(2)}<0$ and as $\operatorname{sgn}\left(a_{1} v_{1_{(2)}}\right)=\operatorname{sgn}\left(a_{2} v_{2_{(2)}}\right)$, we can assure $\left|u_{(2)}\right|=$ $\left|a_{1}\right|\left|v_{1_{(2)}}\right|+\left|a_{2}\right|\left|v_{2_{(2)}}\right|>\left|x_{1}\right|\left|v_{1_{(2)}}\right|+\left|x_{2}\right|\left|v_{2_{(2)}}\right|=\left|v_{(2)}\right|$. So we get $z_{(2)}^{\prime \prime}<0$, which is again a contradiction.

(2) If $\sigma=-1$, we have that $\left|u_{(1)}\right|>\left|v_{(1)}\right|$ and $\left|u_{(3)}\right|>\left|v_{(3)}\right|$.

(a) If $\mathbf{v}=(+, ?,-)$, then $z_{(3)}=0$. The case $u_{(3)}<0$ leads to $z_{(3)}^{\prime \prime}<0$, which is a contradiction; while from $u_{(3)}>0$ we deduce that $u_{(1)}<0$, and as $\operatorname{sgn}\left(a_{1} v_{1_{(1)}}\right)=$ $\operatorname{sgn}\left(\sigma a_{2} v_{2_{(1)}}\right)$, we can assure $\left|u_{(1)}\right|=\left|a_{1}\right|\left|v_{1_{(1)}}\right|+\left|a_{2}\right|\left|\sigma v_{2_{(1)}}\right|>\left|x_{1}\right|\left|v_{1_{(1)}}\right|+\left|x_{2}\right|\left|\sigma v_{2_{(1)}}\right|=$ $\left|v_{(1)}\right|$ we obtain $z_{(1)}^{\prime \prime}<0$, yielding once more a contradiction.

(b) If $\mathbf{v}=(-, ?,+)$, then $z_{(1)}=0$. If the inequality $u_{(1)}<0$ holds, then $z_{(1)}^{\prime \prime}<0$, yielding a contradiction. If $u_{(1)}>0$, then $u_{(3)}<0$, and as $\left|u_{(3)}\right|=\left|a_{2}\right|\left|\sigma v_{2_{(3)}}\right|>\left|x_{2}\right|\left|\sigma v_{2_{(3)}}\right|=\left|v_{(3)}\right|$ we derive $z_{(3)}^{\prime \prime}<0$, which is also a contradiction.

This proves that there is no factorization of $s$ with length between $\ell(\mathbf{z})$ and $\ell\left(\mathbf{z}^{\prime}\right)$.

\section{REFERENCES}

[1] A. Assi, P. A. García-Sánchez, Numerical semigroups and applications, RSME Springer series 1, Springer, Switzerland, 2016.

[2] T. Barron, C. O'Neill, R. Pelayo, On dynamic algorithms for factorization invariants in numerical monoids, to appear in Math of Comp. arXiv:1507.07435

[3] C. Bowles, S. T. Chapman, N. Kaplan and D. Reiser, On Delta Sets of Numerical Monoids, J. Algebra Appl.5 (2006), 1-24.

[4] S. Colton, N. Kaplan, The Realization Problem for Delta Sets of Numerical Semigroups, to appear in J. Commut. Algebra. arXiv:1503.08496.

[5] S. T. Chapman, R. Hoyer and N. Kaplan, Delta Sets of Numerical Monoids are Eventually Periodic, Aequationes Math. 77(2009), 273-279. 
[6] S. T. Chapman, P. A. García-Sánchez, D. Llena, A. Malyshev, D. Steinberg, On the Delta set and the Betti elements of a BF-monoid, Arab. J. Math. 1 (2012), 53-61.

[7] M. Delgado, P.A. García-Sánchez, and J. Morais, NumericalSgps, A package for numerical semigroups, Version 1.0.1 dev (2015), (Refereed GAP package), http://www.gap-system.org/Packages/numericalsgps.html

[8] The GAP Group, GAP - Groups, Algorithms, and Programming, Version 4.7.5; 2014, (http://www.gap-system.org).

[9] J.I. García-García, M.A. Moreno-Frías, A Vigneron-Tenorio, Computation of Delta sets of numerical monoids, Monatsh. Math. 178-3 (2015), 457-472.

[10] P.A. García-Sánchez, D. Llena, A. Moscariello, Delta sets for numerical semigroups with embedding dimension three. arXiv:1504.02116. To Appear in Forum Math.

[11] P.A. García-Sánchez, C. O'Neill, G. Webb, On the computation of factorization invariants for affine semigroups, arXiv:1504.02998.

[12] P. A. García-Sánchez, I. Ojeda, J.C. Rosales, Affine semigroups having a unique Betti element, J. Algebra Appl. 12 (2013), 1250177 (11 pages).

[13] A. Geroldinger, On the arithmetic of certain not integrally closed noetherian integral domains, Comm. Algebra 19(1991), 685-698.

[14] J. C. Rosales, P. A. García-Sánchez, Numerical Semigroups, Developments in Mathematics 20, Springer New York (2009).

Departamento de Álgebra and IEMath-GR, Universidad de Granada, 18071 Granada, España

E-mail address: pedro@ugr.es

Departamento de Matemáticas, Universidad de Almería, 04120 Almería, España

E-mail address: dllena@ual.es

Dipartimento di Matematica, Universit di Pisa, Largo Bruno Pontecorvo 5, 56127 Pisa, Italy.

E-mail address: moscariello@mail.dm.unipi.it 